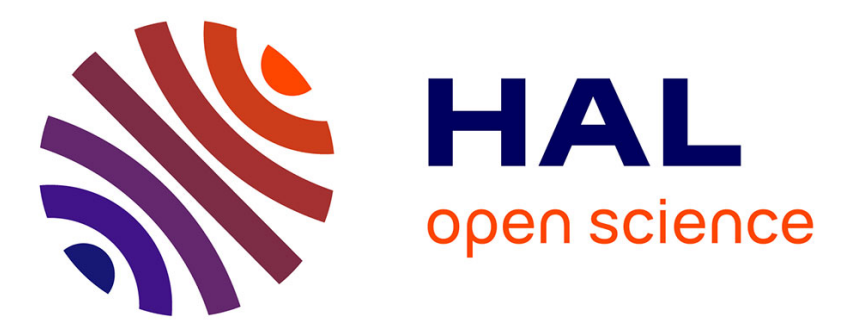

\title{
Space Shift Keying (SSK) Modulation With Partial Channel State Information: Optimal Detector and Performance Analysis Over Fading Channels \\ Marco Di Renzo, Harald Haas
}

\section{- To cite this version:}

Marco Di Renzo, Harald Haas. Space Shift Keying (SSK) Modulation With Partial Channel State Information: Optimal Detector and Performance Analysis Over Fading Channels. IEEE Transactions on Communications, 2010, 58 (11), pp. 3196-3210. hal-00546998

\section{HAL Id: hal-00546998 \\ https://hal.science/hal-00546998}

Submitted on 15 Dec 2010

HAL is a multi-disciplinary open access archive for the deposit and dissemination of scientific research documents, whether they are published or not. The documents may come from teaching and research institutions in France or abroad, or from public or private research centers.
L'archive ouverte pluridisciplinaire HAL, est destinée au dépôt et à la diffusion de documents scientifiques de niveau recherche, publiés ou non, émanant des établissements d'enseignement et de recherche français ou étrangers, des laboratoires publics ou privés. 


\title{
Space Shift Keying (SSK) Modulation with Partial Channel State Information: Optimal Detector and Performance Analysis over Fading Channels
}

\author{
Marco Di Renzo, Member, IEEE, and Harald Haas, Member, IEEE
}

\begin{abstract}
Space Shift Keying (SSK) modulation is a new and recently proposed transmission technology for Multiple-InputMultiple-Output (MIMO) wireless systems, which has been shown to be a promising low-complexity alternative to several state-of-the-art MIMO schemes. So far, only optimal or heuristic transceivers with Full Channel State Information (F-CSI) at the receiver have been investigated, and their performance analyzed over fading channels. In this paper, we develop and study the performance of the optimal Maximum-Likelihood (ML) detector with unknown phase reference at the receiver (i.e., Partial-CSI, P-CSI, knowledge). A very accurate analytical framework for the analysis and optimization of this novel detector over generically correlated and non-identically distributed Nakagami- $m$ fading channels is proposed, and its performance compared to the optimal receiver design with F-CSI. Numerical results will point out that: i) the performance of SSK modulation is significantly affected by the characteristics of fading channels, e.g., channel correlation, fading severity, and, particularly, power imbalance among the transmit-receive wireless links, and ii) unlike ordinary modulation schemes, there is a substantial performance loss when the receiver cannot exploit the phase information for optimal receiver design. This latter result highlights the importance of accurate and reliable channel estimation mechanisms for the efficient operation of SSK modulation over fading channels. Analytical frameworks and theoretical findings will also be substantiated via Monte Carlo simulations.
\end{abstract}

Index Terms-Spatial modulation (SM), space shift keying (SSK) modulation, multiple-input-single-output (MISO) systems, sub-optimal detector, performance analysis, correlated fading channels.

\section{INTRODUCTION}

$\mathbf{S}$ PACE modulation is a recently proposed wireless transmission technique for Multiple-Input-Multiple-Output (MIMO) wireless systems [1]-[4], which relies on the location-specific properties of the wireless channel to convey information (see, e.g., [5]). Along the history, this concept has been termed in different ways with slightly different meanings.

Paper approved by G. Bauch, the Editor for MIMO, Coding and Relaying of the IEEE Communications Society. Manuscript received October 5, 2009; revised February 8, 2010.

This paper was presented in part at the IEEE Sarnoff Symposium, Princeton, NJ, USA, April 2010.

M. Di Renzo is with L2S, UMR 8506 CNRS - SUPELEC - Univ. ParisSud, 3 rue Joliot-Curie, 91192 Gif-sur-Yvette CEDEX (Paris), France (email: marco.direnzo@1ss.supelec.fr).

H. Haas is with The University of Edinburgh, College of Science and Engineering, School of Engineering, Institute for Digital Communications (IDCOM), Alexander Graham Bell Building, King's Buildings, Mayfield Road, Edinburgh, EH9 3JL, Scotland, United Kingdom (UK) (e-mail: h.haas@ed.ac.uk).

Digital Object Identifier 10.1109/TCOMM.2010.091710.090598
In [1], the idea of exploiting the differences in the signals received from different antennas to distinguish the information messages was described for the first time. The method was called Space Shift Keying (SSK) modulation, and two different approaches were proposed: i) a first one with reduced data rate and receiver complexity, where the information to be sent is encoded only on the spatial positions of the transmitantennas, and ii) a second one with higher date rate and receiver complexity, where the information bits are encoded on both spatial and signal constellation diagrams ${ }^{1}$. However, the idea in [1] was useful for a simple $2 \times 1$ MIMO system and still suffered from the main issues of conventional MIMO schemes, i.e., Inter-Channel Interference (ICI), Inter-Antenna Synchronization (IAS), and multiple Radio Frequency (RF) chains at the transmitter [4]. In [2], the principle of using the positions of the transmit-antennas as a source of information was exploited to accomplish a form of multiplexing in the spatial domain, and the method was called Orthogonal SpatialDivision Multiplexing (OSDM). In [3] and [4], the idea in [1] has been recently extended to conceive two new modulation schemes that can be used for MIMO systems with arbitrary transmit- and receive-antennas, and completely avoid ICI, IAS, as well as multiple RF chains. As a matter of fact, although the schemes in [3] and [4] are based on the second and first approach proposed in [1], respectively, they show a fundamental difference: in both cases, a single transmitantenna can radiate power at any time-instant. The MIMO scheme in [3] has been called Spatial Modulation (SM), while in [4] the authors have retained the name SSK modulation. Broadly speaking, the method proposed in [4] is a special instance of the SM scheme introduced in [3], which can tradeoff receiver complexity for data rate (see [4] for details). Throughout this paper, we adopt the term SM to refer to the modulation and coding method described in [3], while we use the term SSK modulation to refer to the special case of SM described in [4], where the information bits are encoded only on the spatial positions of the transmit-antennas.

Recent research efforts have shown that the space modulation paradigm can be a very promising candidate to the design of low-complexity modulation schemes and transceiver architectures for MIMO systems over fading channels (see, e.g., [3], [4], [8] and references therein). In particular, it has been shown that SSK modulation and SM can offer better performance

\footnotetext{
${ }^{1}$ The concept of spatial constellation diagram has been introduced for the first time in [4], and has been further elaborated in [6], [7].
} 
than other state-of-the-art MIMO communication systems, e.g., V-BLAST (Vertical Bell Laboratories Layered SpaceTime) [9], Alamouti [10], and Amplitude Phase Modulation (APM) schemes [3], [4], [8]. Furthermore, these performance gains are obtained with a significant reduction in receiver complexity and system design.

The underlying and fundamental principle of space modulation is twofold: i) at the transmitter, a one-to-one mapping of information bits to transmit-antennas, thus allowing the spatial position of the antennas to convey information, and ii) at the receiver, the exploitation, due to the locationspecific properties of wireless fading channels [5], of distinct multipath profiles along any transmit-receive wireless link for data detection. Moving from these basic working principles, the following contributions are available in the literature as far as optimal and sub-optimal receiver design is concerned. i) In [3], a heuristic receiver for SM is proposed, and its performance analyzed over correlated Rayleigh and Rice fading channels. The performance analysis of this detector over correlated Nakagami- $m$ fading channels can be found in [11]. ii) In [8], the optimal detector for SM with Full Channel State Information (F-CSI) at the receiver is developed, and its performance studied over uncorrelated Rayleigh fading channels. iii) Moving from [8], the optimal detector for SSK modulation is introduced in [4], and its performance analyzed for uncoded and coded systems. Moreover, the authors study the effect of imperfect channel estimates when the receiver is still designed to have F-CSI. However, the results are obtained by using Monte Carlo simulations and by assuming that the estimation errors contribute to additive noise only. iv) Moving from [1], an optimization framework to allow more than one transmit-antenna at a time to convey information is introduced in [6], and some performance improvements, due to the optimal constellation design, are shown. The optimal detector with F-CSI is always adopted. v) Motivated by the observation that channel correlation is one of the fundamental practical issues to be taken into account to allow the adoption of SM over realistic propagation environments, a novel SM scheme named Trellis Coded Spatial Modulation (TCSM) is introduced in [7] with the aim to exploit trellis coding to reduce, on average, the effect of fading spatial correlation. vi) Finally, in [12] we have recently developed a very accurate analytical framework to study the performance of SSK modulation over correlated Nakagami- $m$ fading channels adopting a Maximum-Likelihood (ML) detector with F-CSI at the receiver, and highlighted that the characteristics of fading channels can remarkably affect the performance of the space modulation principle.

By carefully reviewing all the above mentioned contributions, we can recognize that all of them consider either optimal or sub-optimal receiver architectures with F-CSI available at the detector. Although the design and analysis of F-CSI-assisted transceivers are of paramount importance to get fundamental insights about receiver operations, as well as to get lower bounds on the achievable performance of them, there are several circumstances in which the channel fading might be sufficiently rapid. Perfect knowledge of CSI at the receiver can, therefore, not be assumed. This, clearly, influences the optimal receiver design [13, Ch. 14]. This con- sideration is theoretically and practically relevant especially for space modulation where, unlike conventional modulation schemes where the information is conveyed by a modulated signal, the information is embedded into the location-specific characteristics of the wireless channel [1], [5]. In particular, it can be easily predicted that, in a wireless system adopting the space modulation paradigm, neglecting part of the locationspecific channel fingerprint along any transmit-receive path may introduce some performance losses. Motivated by these considerations, the main aim of this paper is to quantitatively analyze the performance degradation that is obtained when the detector cannot exploit the channel phase information for optimal decision-making operations.

More precisely, in this paper we develop the optimal ML detector with unknown phase reference at the receiver, thus yielding a Partial-CSI- (P-CSI) assisted receiver design, as opposed to F-CSI optimal detectors available in the literature so far. In particular, we will focus our attention on a $N_{t} \times 1$ MISO (Multiple-Input-Single-Output) SSKbased system setup ( $N_{t}$ is the number of antennas at the transmitter). The rationales for this choice are as follows: i) SSK modulation offers a simpler receiver design than SM and exploits the most important feature of space modulation, i.e., data information is encoded into the spatial position of the transmit-antennas only; ii) with respect to other MIMO concepts, e.g., V-BLAST, SSK modulation can be used when the number of receive-antennas is smaller than the number of transmit-antennas: the $N_{t} \times 1$ MISO setup may find important applications to low-complexity and low-cost downlink settings and operations, where it is more economical to add equipment to base stations rather than to remote mobile units; and iii) this system setup allows us to keep the analytical development at a moderate level, but still offers insightful information about the receiver performance over realistic propagation conditions. Due to the analytical complexity of the problem and to space constraints, the analysis of a general system setup encompassing SM and multiple receive-antennas is left to future research.

More specifically, the main contributions of this paper are as follows: i) the optimal receiver with unknown phase reference is derived, ii) a sub-optimal, and asymptotically tight (for high Signal-to-Noise-Ratios, SNRs), version of it is proposed, iii) when $N_{t}=2$, an asymptotically tight approximation for computing the Average Bit Error Probability (ABEP) over generically correlated and non-identically distributed Nakagami- $m$ fading channels is introduced, and its accuracy is validated for various system settings, and iv) this latter framework is generalized to $N_{t}>2$ by exploiting two upper bounds recently introduced in [12]. Our theoretical and numerical analysis will highlight the following important outcomes. i) The optimal receiver design with P-CSI results in a substantial performance loss with respect to the optimal detector with $\mathrm{F}-$ CSI. This result is in net contrast with ordinary modulation schemes in which the performance loss of a receiver with and without phase information is limited to a few $\mathrm{dB}$. For example, BPSK (Binary Phase Shift Keying) and DBPSK (Differential BPSK) receivers differ less than $1 \mathrm{~dB}$ over Additive White Gaussian Noise (AWGN) channels [13, Fig. 5.2.12], and approximately $3 \mathrm{~dB}$ over Rayleigh fading channels [13, Fig. 
14.3.1 $]^{2}$. ii) The performance of both F-CSI- [12] and PCSI-assisted detectors strongly depends on channel fading statistics, and, particularly, on the power imbalance among the transmit-receive wireless links. iii) Accordingly, accurate and reliable channel estimation mechanisms appear to be of paramount importance for the adoption and efficient operation of the space modulation paradigm in realistic operating environments.

The remainder of the manuscript is organized as follows. In Section II, system and channel models are introduced. In Section III, the optimal detector with P-CSI, along with a lowcomplexity implementation of it for high SNRs, is proposed. In Section IV, the analytical framework for performance analysis of $2 \times 1$ MISO systems over independent and generically correlated Nakagami- $m$ fading channels is developed. In Section V, the framework in Section IV is extended to multiple $\left(N_{t}>2\right)$ transmit-antennas. In Section VI, numerical and simulation results are shown to substantiate the accuracy of the analytical framework and to compare F-CSI- and P-CSIassisted detectors. Finally, Section VII concludes the paper.

\section{SySTEM MOdeL}

Let us consider a generic $N_{t} \times 1$ MISO system, with $N_{t}$ being the number of antennas at the transmitter. According to [4], the detection process of SSK modulation can be cast in terms of a general $N_{t}$-hypothesis decision problem in AWGN [17, Sec. 4.2, pp. 257], when conditioning upon fading channel statistics. More specifically, SSK modulation works as follows: i) the transmitter encodes blocks of $\log _{2}\left(N_{t}\right)$ data bits into the index of a single transmit-antenna, which is switched on for data transmission while all the other antennas are kept silent, and ii) the receiver solves a $N_{t}$-hypothesis detection problem to estimate the transmit-antenna that is not idle, which results in the estimation of the unique sequence of bits emitted by the encoder. Throughout this paper, the unique block of bits encoded into the index of the $i$-th transmit-antenna is called "message" and is denoted by $\left\{u_{i}\right\}_{i=1}^{N_{t}}$. The $N_{t}$ messages are assumed to be emitted with equal probability by the encoder. Moreover, the related transmitted signal is denoted by $\left\{s_{i}(\cdot)\right\}_{i=1}^{N_{t}}$. It is implicitly assumed with this notation that, if $u_{i}$ is transmitted, the analog signal $s_{i}(\cdot)$ is emitted by the $i$-th transmit-antenna while all the other transmit-antennas radiate no power.

\section{A. Notation}

Let us briefly introduce the main notation used in what follows. i) We adopt a complex-envelope signal representation. ii) $j=\sqrt{-1}$ is the imaginary unit. iii) $(\cdot)^{*}$ denotes

\footnotetext{
${ }^{2}$ Let us emphasize that differential detection schemes require the knowledge of neither the channel envelopes nor the channel phases, but they extract this information from a previously transmitted signal. On the contrary, our detector still requires the knowledge of the channel envelopes. So, with this example, we want to simply emphasize that for conventional modulation schemes there are methods that avoid channel estimation at the receiver, while this problem is still completely unexplored in the recently proposed space modulation systems. Furthermore, since the wireless channel acts as a modulation unit for SSK and SM, the development of either differential or non-coherent schemes seems to be a non-trivial task. Finally, we also note that differential schemes are available for multiple-transmit antennas as well, and they always incur in a $3 \mathrm{~dB}$ performance loss with respect to coherent solutions with full channel knowledge (see, e.g. [14], [15], and [16, Sec. III-H]).
}

complex-conjugate. iv) $(x \otimes y)(t)=\int_{-\infty}^{+\infty} x(\xi) y(t-\xi) d \xi$ is the convolution of signals $x(\cdot)$ and $y(\cdot)$. v) $|\cdot|^{2},|\cdot|$, and $\angle$. denote square absolute value, absolute value and phase angle of a complex vector, respectively. vi) $\mathrm{E}_{\mathrm{A}}\{\cdot\}$ is the expectation operator computed over the Random Variable (RV) A. vii) $\operatorname{Re}\{\cdot\}$ denotes the real part operator. viii) $\operatorname{Pr}\{\cdot\}$ denotes probability. ix) $\rho_{\mathrm{AB}}$ denotes the correlation coefficient of RVs A and B. x) $Q(\cdot, \cdot)$ is the Marcum Q-function [18], [19, Eq. (3)]. xi) $\hat{u}$ denotes the message estimated at the receiver-side. xii) $E_{u}$ is the average energy transmitted by each antenna that emits a non-zero signal. xiii) $T_{u}$ denotes the signaling interval of each information message $\left\{u_{i}\right\}_{i=1}^{N_{t}}$. xiv) The noise at the receiver input is denoted by $n(\cdot)$, and is assumed to be AWG-distributed, with both real and imaginary parts having a double-sided power spectral density equal to $N_{0}$. xv) $\left\{s_{i}(t)\right\}_{i=1}^{N_{t}}=\sqrt{E_{u}} w(t)$ with $w(\cdot)$ denoting the unit-energy (i.e., $\int_{-\infty}^{+\infty}|w(t)|^{2} d t=1$ ) elementary baseband waveform for each transmission. xvi) $\operatorname{erfc}(\cdot)$ is the complementary error function [20, Eq. (7.1.2))]. xvii) $\Gamma(\cdot)$ is the Gamma function [20, Eq. (6.1.1)]. xviii) $I_{\nu}(\cdot)$ is the modified Bessel function of first kind and order $\nu$ [20, Ch. 9)]. xix) $G_{p, q}^{m, n}\left(. \mid \begin{array}{c}\left(a_{p}\right) \\ \left(b_{q}\right)\end{array}\right)$ is the Meijer-G function defined in [21, Ch. 8, pp. 519]. $\mathrm{xx}) \delta(\cdot)$ is the Dirac delta function. $\mathrm{xxi}$ ) $L_{n}^{\nu}(\cdot)$ is the Generalized Laguerre polynomial in [20, Eq. (22.2.12)]. xxii) $(\cdot)_{n}$ is the Pochhammer symbol, which is defined as $(a)_{n}=\Gamma(a+n) / \Gamma(a)$. xxiii) (.) denotes the binomial coefficient. xxiv) For ease of notation, we define $\bar{\gamma}=\sqrt{E_{u} / N_{0}}$.

\section{B. Channel Model}

We consider a frequency-flat slowly-varying fading channel model, with fading envelopes distributed according to a Nakagami- $m$ distribution [22]. Moreover, we assume generically correlated and non-identically distributed fading parameters in this manuscript. In particular:

- $\left\{h_{i}(t)\right\}_{i=1}^{N_{t}}=\beta_{i} \exp \left(j \varphi_{i}\right) \delta\left(t-\tau_{i}\right)$ is the channel impulse response of the $i$-th wireless link, where $\left\{\beta_{i}\right\}_{i=1}^{N_{t}}$, $\left\{\varphi_{i}\right\}_{i=1}^{N_{t}}$, and $\left\{\tau_{i}\right\}_{i=1}^{N_{t}}$ denote gain, phase, and delay, respectively. Moreover, $\left\{\alpha_{i}\right\}_{i=1}^{N_{t}}=\beta_{i} \exp \left(j \varphi_{i}\right)$ denotes the channel complex-gain.

- $\left\{\tau_{i}\right\}_{i=1}^{N_{t}}$ is assumed to be independent and uniformly distributed in $\left[0, T_{m}\right)$, but known at the receiver, i.e., perfect time-synchronization is considered. Similar to [3], [4], [8], we assume $\tau_{1} \cong \tau_{2} \cong \ldots \cong \tau_{N_{t}}$, which is a realistic hypothesis when the distance between transmitter and receiver is much larger than the spacing between the transmit-antennas, and, to a first-order, the signals transmitted by the antennas differ only in phase [23, Eq. (7.24)]. The assumptions of perfect time-synchronization at the receiver and almost equal propagation delays allow us to neglect $\left\{\tau_{i}\right\}_{i=1}^{N_{t}}$ from our notation and subsequent analysis.

- $\left\{\varphi_{i}\right\}_{i=1}^{N_{t}}$ is assumed to be independent and uniformly distributed in $[0,2 \pi)$.

- The channel envelopes, $\left\{\beta_{i}\right\}_{i=1}^{N_{t}}$, are assumed to be distributed according to a multivariate Nakagami- $m$ distribution. In particular, when $N_{t}=2$ various joint 


$$
D_{i}\left(\varphi_{i}\right)=\exp \left[\frac{1}{N_{0}} \operatorname{Re}\left\{\int_{T_{u}} r(t) \tilde{s}_{i}^{*}(t) d t\right\}-\frac{1}{2 N_{0}} \int_{T_{u}} \tilde{s}_{i}(t) \tilde{s}_{i}^{*}(t) d t\right]
$$

Probability Density Functions (PDFs), $\left\{f_{\beta_{i} \beta_{j}}(\cdot)\right\}_{i \neq j=1}^{N_{t}}$, will be considered in Section IV, such as [24, Eq. (6.1)] and [25, Eq. (12)]. In particular, [25, Eq. (12)] is the most general formulation of the PDF of bivariate Nakagami$m$ RVs with arbitrary correlation and fading parameters. By using the tight upper bounds recently introduced in [12], we will see that, to analyze the performance of the $N_{t} \times 1$ MISO system at hand, only the joint PDF of pairs of Nakagami- $m$ RVs is required. Further details about the rationale and generality of considering the Nakagami- $m$ fading channel model (instead of other channel models) can be found in [12] and are here omitted due to space constraints.

- The fading parameters of the $i$-th wireless link are denoted by $\left\{m_{i}\right\}_{i=1}^{N_{t}}$ and $\left\{\Omega_{i}\right\}_{i=1}^{N_{t}}=\mathrm{E}_{\beta_{i}}\left\{\beta_{i}^{2}\right\}$.

\section{Optimal ML Detector With P-CSI}

Let $\left\{u_{l}\right\}_{l=1}^{N_{t}}$ be the actual transmitted message ${ }^{3}$. Moving from the system and channel models in Section II, the signals after propagation through the wireless fading channel are $\left\{\tilde{s}_{l}(t)\right\}_{l=1}^{N_{t}}=\left(s_{l} \otimes h_{l}\right)(t)=\beta_{l} \exp \left(j \varphi_{l}\right) s_{l}(t)$, and the received signal can be written as follows:

$$
r(t)=\tilde{s}_{l}(t)+n(t) \quad \text { if } u_{l} \text { is sent }
$$

which is a general $N_{t}$-hypothesis detection problem [17, Sec. 4.2, pp. 257], [24, Sec. 7.1].

From (2), the optimal ML detector with unknown phase reference (P-CSI) and perfect time-synchronization at the receiver is as follows [24, Sec. 7.4]:

$$
\begin{aligned}
\hat{u}= & \underset{\left\{u_{i}\right\}_{i=1}^{N_{t}}}{\arg \max }\left\{\ln \left[D_{i}\right]\right\} \\
= & \underset{\left\{u_{i}\right\}_{i=1}^{N_{t}}}{\arg \max }\left\{\ln \left[\mathrm{E}_{\varphi_{i}}\left\{D_{i}\left(\varphi_{i}\right)\right\}\right]\right\} \\
= & \underset{\left\{u_{i}\right\}_{i=1}^{N_{t}}}{\arg \max }\left\{\ln \left[\frac{1}{2 \pi} \int_{0}^{2 \pi} D_{i}\left(\phi_{i}\right) d \phi_{i}\right]\right\}
\end{aligned}
$$

where $\left\{D_{i}(\cdot)\right\}_{i=1}^{N_{t}}$ are the decision metrics conditioned upon the channel phases $\left\{\varphi_{i}\right\}_{i=1}^{N_{t}}$, which are defined in (1) on top of this page.

Let us emphasize that, with respect to the F-CSI-assisted detector in [4], the decision metrics $\left\{D_{i}(\cdot)\right\}_{i=1}^{N_{t}}$ in (1) need to be averaged over the distribution of the channel phases, thus making the detector blind to them. On the contrary, in [4] the channel phases need to be provided to the detector via suitable channel estimation algorithms. Accordingly, the overall complexity (by including also the complexity of the channel estimator) of the receiver in (3) will be significantly reduced.

\footnotetext{
${ }^{3}$ In order to avoid any confusion with the adopted notation, let us emphasize that the subscript $l$ denotes the actual message that is transmitted, while the subscript $i$ denotes the (generic) $i$-th message that is tested by the detector to solve the $N_{t}$-hypothesis detection problem. More specifically, for each signaling interval, $l$ is fixed, while $i$ can take different values at the detector.
}

By using the definitions in Section II-A, (2) and (1) can be explicitly re-written as follows, respectively:

$$
\begin{gathered}
r(t)=\sqrt{E_{u}} \beta_{l} \exp \left(j \varphi_{l}\right) w(t)+n(t) \\
D_{i}\left(\varphi_{i}\right)=\exp \left[\frac{\sqrt{E_{u}} \beta_{i}}{N_{0}}|\bar{r}| \cos \left(\varphi_{i}-\angle \bar{r}\right)-\frac{E_{u} \beta_{i}^{2}}{2 N_{0}}\right]
\end{gathered}
$$

where we have defined $\bar{r}=\int_{T_{u}} r(t) w^{*}(t) d t=$ $|\bar{r}| \exp (j \angle \bar{r})$.

By averaging $\left\{D_{i}(\cdot)\right\}_{i=1}^{N_{t}}$ in (5) over the distribution of $\left\{\varphi_{i}\right\}_{i=1}^{N_{t}}$ using analytical steps similar to [17, pp. 339, Eq. (366), Eq. (367)], and then computing the logarithm of the obtained result we obtain:

$$
\bar{D}_{i}=\ln \left[D_{i}\right]=\ln \left[I_{0}\left(\frac{\sqrt{E_{u}} \beta_{i}}{N_{0}}|\bar{r}|\right)\right]-\frac{E_{u} \beta_{i}^{2}}{2 N_{0}}
$$

which, along with (3), yields the optimal detector with P-CSI at the receiver and agrees, e.g., with [24, Eq. (7.24)]. Note that the detector will be successful in detecting the transmitted message, i.e., $\hat{u}=u_{l}$, if and only if $\max _{i=1,2, \ldots, N_{t}}\left\{\bar{D}_{i}\right\}=\bar{D}_{l}$.

The analysis of the detector in (6) is quite cumbersome due to the Bessel function $I_{0}(\cdot)$ that needs to be computed. A simpler and asymptotically (for high SNRs) equivalent detector can be obtained by recognizing that $\ln \left[I_{0}(|x|)\right] \cong|x|$ when $|x| \gg 1$. By exploiting this asymptotic approximation, the decision metric in (6) simplifies as follows ${ }^{4}$ :

$$
\bar{D}_{i}=\sqrt{E_{u}} \beta_{i}|\bar{r}|-\frac{E_{u} \beta_{i}^{2}}{2}
$$

where irrelevant constants have also been neglected.

By carefully looking at (7), we can observe that $\left\{\bar{D}_{i}\right\}_{i=1}^{N_{t}}$ is very similar to the heuristic detector used in [3, Eq. (3)] for estimating the antenna index ${ }^{5}$. In particular, they differ for the bias factor $E_{u} \beta_{i}^{2} / 2$, which allows (7) to cope with the assumption of constrained channels remarked in [8]. In other words, the detector in [3, Eq. (3)] is not completely heuristic, but can be interpreted, apart from the bias term discussed above, as a high SNR approximation of the optimal detector with unknown phase reference at the receiver. This is an understandable outcome when it is observed that the detector in [3, Eq. (3)] is used to make the estimation process of the antenna index independent from the estimation process of the data transmitted by each antenna.

In the next two sections, we will develop an accurate analytical framework for computing the ABEP of the detector in (7). We will realize that, while the detectors in (6) and

\footnotetext{
${ }^{4}$ Note that if the receiver is equipped with multiple antennas, the optimal ML detector will exploit them to get diversity gains. In particular, in this latter scenario, a detector similar to [24, Sec. 7.3 , pp. 166] could be obtained by using the same analytical steps as those already used to compute (7). A similar result has already been obtained in [4] and [8] for the optimal detector with F-CSI at the receiver. However, in this paper we have decided not to consider this system setup due to the complexity of computing the ABEP for multiple receive-antennas, and have postponed its analysis to a future contribution.

${ }^{5}$ Note, however, that in [3, Eq. (3)] the detector is still assumed to have F-CSI, as highlighted in [8, Eq. (2)].
} 


$$
\left.\mathrm{P}_{\mathrm{E}}\left(h_{1}, h_{2}\right)\right|_{u_{1}}= \begin{cases}\operatorname{Pr}\left\{\left|\sqrt{E_{u}} \beta_{1} \exp \left(j \varphi_{1}\right)+\bar{n}\right|<\frac{\sqrt{E_{u}}}{2}\left(\beta_{1}+\beta_{2}\right)\right\} & \text { if } \beta_{1} \geq \beta_{2} \\ \operatorname{Pr}\left\{\left|\sqrt{E_{u}} \beta_{1} \exp \left(j \varphi_{1}\right)+\bar{n}\right|>\frac{\sqrt{E_{u}}}{2}\left(\beta_{1}+\beta_{2}\right)\right\} & \text { if } \beta_{1}<\beta_{2}\end{cases}
$$

$$
\begin{aligned}
\mathrm{P}_{\mathrm{E}}\left(h_{1}, h_{2}\right) & =\left[\frac{1}{2}-\frac{1}{2} Q\left(\frac{\sqrt{E_{u}} \beta_{1}}{\sqrt{N_{0}}}, \frac{\sqrt{E_{u}}\left(\beta_{1}+\beta_{2}\right)}{2 \sqrt{N_{0}}}\right)+\frac{1}{2} Q\left(\frac{\sqrt{E_{u}} \beta_{2}}{\sqrt{N_{0}}}, \frac{\sqrt{E_{u}}\left(\beta_{1}+\beta_{2}\right)}{2 \sqrt{N_{0}}}\right)\right] \cdot \operatorname{Pr}\left\{\beta_{1} \geq \beta_{2}\right\} \\
& +\left[\frac{1}{2}-\frac{1}{2} Q\left(\frac{\sqrt{E_{u}} \beta_{2}}{\sqrt{N_{0}}}, \frac{\sqrt{E_{u}}\left(\beta_{1}+\beta_{2}\right)}{2 \sqrt{N_{0}}}\right)+\frac{1}{2} Q\left(\frac{\sqrt{E_{u}} \beta_{1}}{\sqrt{N_{0}}}, \frac{\sqrt{E_{u}}\left(\beta_{1}+\beta_{2}\right)}{2 \sqrt{N_{0}}}\right)\right] \cdot \operatorname{Pr}\left\{\beta_{1}<\beta_{2}\right\}
\end{aligned}
$$

$$
Q(a, b) \leq \frac{I_{0}(a b)}{\exp (a b)}\left\{\exp \left[-\frac{(b-a)^{2}}{2}\right]+a \sqrt{\frac{\pi}{2}} \operatorname{erfc}\left(\frac{b-a}{\sqrt{2}}\right)\right\} \cong \underbrace{\frac{1}{\sqrt{2 \pi a b}} \exp \left[-\frac{(b-a)^{2}}{2}\right]}_{P_{1}(a, b)}+\underbrace{\frac{1}{2} \sqrt{\frac{a}{b}} \operatorname{erfc}\left(\frac{b-a}{\sqrt{2}}\right)}_{P_{2}(a, b)}
$$

$$
\begin{aligned}
1-Q(a, b) & \geq \frac{I_{0}(a b)}{\exp (a b)}\left\{\exp \left(-\frac{a^{2}}{2}\right)-\exp \left[-\frac{(b-a)^{2}}{2}\right]+a \sqrt{\frac{\pi}{2}} \operatorname{erfc}\left(-\frac{a}{\sqrt{2}}\right)-a \sqrt{\frac{\pi}{2}} \operatorname{erfc}\left(\frac{b-a}{\sqrt{2}}\right)\right\} \\
& \cong \underbrace{\frac{1}{\sqrt{2 \pi a b}} \exp \left(-\frac{a^{2}}{2}\right)}_{P_{3}(a, b)}-\underbrace{\frac{1}{\sqrt{2 \pi a b}} \exp \left[-\frac{(b-a)^{2}}{2}\right]}_{P_{1}(a, b)}+\underbrace{\frac{1}{2} \sqrt{\frac{a}{b}} \operatorname{erfc}\left(-\frac{a}{\sqrt{2}}\right)}_{P_{4}(a, b)}-\underbrace{\frac{1}{2} \sqrt{\frac{a}{b}} \operatorname{erfc}\left(\frac{b-a}{\sqrt{2}}\right)}_{P_{2}(a, b)}
\end{aligned}
$$

$$
\begin{aligned}
\mathrm{P}_{\mathrm{E}}\left(h_{1}, h_{2}\right) \cong & \frac{1}{2} \sum_{k=1}^{4}\left[\vartheta_{k} P_{k}\left(\gamma_{1}, \gamma_{1,2}\right) \operatorname{Pr}\left\{\beta_{1} \geq \beta_{2}\right\}\right]+\frac{1}{2} \sum_{k=1}^{2}\left[P_{k}\left(\gamma_{2}, \gamma_{1,2}\right) \operatorname{Pr}\left\{\beta_{1} \geq \beta_{2}\right\}\right] \\
& +\frac{1}{2} \sum_{k=1}^{4}\left[\vartheta_{k} P_{k}\left(\gamma_{2}, \gamma_{1,2}\right) \operatorname{Pr}\left\{\beta_{1}<\beta_{2}\right\}\right]+\frac{1}{2} \sum_{k=1}^{2}\left[P_{k}\left(\gamma_{1}, \gamma_{1,2}\right) \operatorname{Pr}\left\{\beta_{1}<\beta_{2}\right\}\right]
\end{aligned}
$$

$$
\left\{\begin{array}{l}
\left\{P_{k}^{>}(p)\right\}_{p=1}^{2}=\int_{0}^{+\infty} \int_{0}^{\xi_{1}} P_{k}\left(\bar{\gamma} \xi_{p}, \frac{\bar{\gamma}}{2}\left(\xi_{1}+\xi_{2}\right)\right) f_{\beta_{1} \beta_{2}}\left(\xi_{1}, \xi_{2}\right) d \xi_{1} d \xi_{2} \\
\left\{P_{k}^{<}(p)\right\}_{p=1}^{2}=\int_{0}^{+\infty} \int_{0}^{\xi_{2}} P_{k}\left(\bar{\gamma} \xi_{p}, \frac{\bar{\gamma}}{2}\left(\xi_{1}+\xi_{2}\right)\right) f_{\beta_{1} \beta_{2}}\left(\xi_{1}, \xi_{2}\right) d \xi_{1} d \xi_{2}
\end{array}\right.
$$

(7) have been obtained by using well-known techniques, the computation of the ABEP over fading channels will require novel analytical approaches to deal with the specific signal structure of SSK modulation. To efficiently handle the complexity of this problem, a tight approximation will be proposed. Moreover, due to space constraints, in this paper we will only consider the detector in (7). The study of the tightness of (6) and (7) for high SNRs can be found in [26].

\section{ABEP OVER NAKAGAMI- $m$ FADING ChanNELS: THE $2 \times 1$ MISO SETUP}

Let us consider $N_{t}=2$. From the decision rule in (3), the probability of error, $\mathrm{P}_{\mathrm{E}}(\cdot, \cdot)$, of the detection process (i.e., the detection of the index of the transmit-antenna), when conditioning upon the channel impulse responses $\left\{h_{i}(\cdot)\right\}_{i=1}^{2}$, can be written as follows:

$$
\begin{aligned}
\mathrm{P}_{\mathrm{E}}\left(h_{1}, h_{2}\right) & =\left.\frac{1}{2} \mathrm{P}_{\mathrm{E}}\left(h_{1}, h_{2}\right)\right|_{u_{1}}+\left.\frac{1}{2} \mathrm{P}_{\mathrm{E}}\left(h_{1}, h_{2}\right)\right|_{u_{2}} \\
& =\frac{1}{2} \operatorname{Pr}\left\{\left.\bar{D}_{1}\right|_{u_{1}}<\left.\bar{D}_{2}\right|_{u_{1}}\right\} \\
& +\frac{1}{2} \operatorname{Pr}\left\{\left.\bar{D}_{2}\right|_{u_{2}}<\left.\bar{D}_{1}\right|_{u_{2}}\right\}
\end{aligned}
$$

where $\left\{\left.\mathrm{P}_{\mathrm{E}}(\cdot, \cdot)\right|_{u_{i}}\right\}_{i=1}^{2}$ and $\left\{\left.\bar{D}_{j}\right|_{u_{i}}\right\}_{i, j=1}^{2}$ denote the probabilities of error and the decision metrics conditioned upon the transmission of messages $\left\{u_{i}\right\}_{i=1}^{2}$, respectively.

\section{A. Conditional BEP - Fixed Channel Realization}

Let us start with the computation of $\left.\mathrm{P}_{\mathrm{E}}(\cdot, \cdot)\right|_{u_{1}}$. By plugging (4) into (7), and after a few algebraic manipulations, we obtain (9) on top of this page, where we have defined $\bar{n}=\int_{T_{u}} n(t) w^{*}(t) d t$. 
Moreover, we can readily recognize that $R_{1}=$ $\left|\sqrt{E_{u}} \beta_{1} \exp \left(j \varphi_{1}\right)+\bar{n}\right|$ is a Rice-distributed $\mathrm{RV}$ having Cumulative Distribution Function $(\mathrm{CDF}), F_{R_{1}}(\cdot)$, equal to $F_{R_{1}}(\xi)=1-Q(s / \sigma, r / \sigma)$ [13, Eq. 2.1.142] with $s=$ $\sqrt{E_{u}} \beta_{1}$ and $\sigma=\sqrt{N_{0}}$. Accordingly, (9) reduces as follows:

$$
\left.\mathrm{P}_{\mathrm{E}}\left(h_{1}, h_{2}\right)\right|_{u_{1}}= \begin{cases}1-Q\left(\frac{\sqrt{E_{u}} \beta_{1}}{\sqrt{N_{0}}}, \frac{\sqrt{E_{u}}\left(\beta_{1}+\beta_{2}\right)}{2 \sqrt{N_{0}}}\right) & \text { if } \beta_{1} \geq \beta_{2} \\ Q\left(\frac{\sqrt{E_{u}} \beta_{1}}{\sqrt{N_{0}}}, \frac{\sqrt{E_{u}}\left(\beta_{1}+\beta_{2}\right)}{2 \sqrt{N_{0}}}\right) & \text { if } \beta_{1}<\beta_{2}\end{cases}
$$

With similar analytical steps, which are here omitted due to space constraints, we can obtain a similar result for $\left.\mathrm{P}_{\mathrm{E}}(\cdot, \cdot)\right|_{u_{2}}$ as follows:

$$
\left.\mathrm{P}_{\mathrm{E}}\left(h_{1}, h_{2}\right)\right|_{u_{2}}= \begin{cases}1-Q\left(\frac{\sqrt{E_{u}} \beta_{2}}{\sqrt{N_{0}}}, \frac{\sqrt{E_{u}}\left(\beta_{1}+\beta_{2}\right)}{2 \sqrt{N_{0}}}\right) & \text { if } \beta_{2} \geq \beta_{1} \\ Q\left(\frac{\sqrt{E_{u}} \beta_{2}}{\sqrt{N_{0}}}, \frac{\sqrt{E_{u}}\left(\beta_{1}+\beta_{2}\right)}{2 \sqrt{N_{0}}}\right) & \text { if } \beta_{2}<\beta_{1}\end{cases}
$$

As a consequence, the error probability in (8) can be written in closed-form as shown in (12) on top of the previous page. In the next sub-sections, we will also show that the probabilities $\operatorname{Pr}\left\{\beta_{1} \geq \beta_{2}\right\}$ and $\operatorname{Pr}\left\{\beta_{1}<\beta_{2}\right\}$ do not need to be actually computed to obtain the ABEP.

\section{B. Tight Approximation for $\mathrm{P}_{\mathrm{E}}(\cdot, \cdot)$}

By carefully looking at (12), we can readily figure out that computing the ABEP, i.e., $\mathrm{ABEP}=\mathrm{E}_{h_{1}, h_{2}}\left\{\mathrm{P}_{\mathrm{E}}\left(h_{1}, h_{2}\right)\right\}=$ $\mathrm{E}_{\beta_{1}, \beta_{2}}\left\{\mathrm{P}_{\mathrm{E}}\left(h_{1}, h_{2}\right)\right\}$, and, therefore, removing the conditioning over the wireless channel statistics, can be quite involving. As a matter of fact, in this specific case we are unable to use well-consolidated tools to re-write the Marcum Q-function in (12) in an equivalent integral form useful for averaging over the distribution of the fading envelopes [27, Eq. (14a), Eq. (14b)]. As a consequence, novel specific communicationtheoretic frameworks seem to be required to understand and analyze the performance of SSK modulation over fading channels.

In this paper, we propose to jointly use an upper and a lower bound, which exploit the results in [19] and are given in a suitable form to solve the problem at hand. In particular, to approximate $\mathrm{P}_{\mathrm{E}}(\cdot, \cdot)$ in (12) we suggest to use the upper bound [19, Eq. (7)] and the lower bound [19, Eq. (12)] for the Marcum Q-function which are shown in (13) and (14) on top of the previous page. To further reduce the analytical complexity, in (13) and (14) we have exploited the approximation in [28, Eq. (8)] for the $I_{0}(\cdot)$ Bessel function.

Then, by plugging (13) and (14) into $(12), \mathrm{P}_{\mathrm{E}}(\cdot, \cdot)$ can be tightly approximated as shown in (15) on top of the previous page, where we have defined $\gamma_{1}=\beta_{1} \sqrt{E_{u} / N_{0}}$, $\gamma_{2}=\beta_{2} \sqrt{E_{u} / N_{0}}, \gamma_{1,2}=(1 / 2)\left(\beta_{1}+\beta_{2}\right) \sqrt{E_{u} / N_{0}}$, and $\left\{\vartheta_{k}\right\}_{k=1}^{4}=\{-1,-1,1,1\}$.

\section{C. $A B E P$}

We observe that the final result in (15) is now expressed in a very convenient form to be averaged over the fading envelopes $\left(\beta_{1}, \beta_{2}\right)$, given that the conditional $\mathrm{BEP}, \mathrm{P}_{\mathrm{E}}(\cdot, \cdot)$, is expressed as the summation of elementary functions. In particular, the
ABEP can be readily written as shown in what follows:

$$
\begin{aligned}
\mathrm{ABEP} & \cong \frac{1}{2} \sum_{k=1}^{4}\left[\vartheta_{k} P_{k}^{>}(1)\right]+\frac{1}{2} \sum_{k=1}^{2}\left[P_{k}^{>}(2)\right] \\
& +\frac{1}{2} \sum_{k=1}^{4}\left[\vartheta_{k} P_{k}^{<}(2)\right]+\frac{1}{2} \sum_{k=1}^{2}\left[P_{k}^{<}(1)\right]
\end{aligned}
$$

where we have defined $\left\{P_{k}^{>}(p)\right\}_{p=1}^{2}=$ $\mathrm{E}_{\beta_{1}, \beta_{2}}\left\{P_{k}\left(\gamma_{p}, \gamma_{1,2}\right) \operatorname{Pr}\left\{\beta_{1} \geq \beta_{2}\right\}\right\} \quad$ and $\quad\left\{P_{k}^{<}(p)\right\}_{p=1}^{2}=$ $\mathrm{E}_{\beta_{1}, \beta_{2}}\left\{P_{k}\left(\gamma_{p}, \gamma_{1,2}\right) \operatorname{Pr}\left\{\beta_{1}<\beta_{2}\right\}\right\}$. Furthermore, each expectation in (16) can be computed from the definition of $\mathrm{E}_{\beta_{1}, \beta_{2}}(\cdot)$, as shown in (17) on top of the previous page.

In the next sub-sections, the integrals in (17) will be computed for independent and generically correlated Nakagami- $m$ fading channels, according to the channel model introduced in Section II-B.

\section{Independent Fading}

Let us analyze the scenario with uncorrelated fading envelopes, i.e., $\rho_{\beta_{1}^{2} \beta_{2}^{2}}=0$. In this case, $f_{\beta_{1}, \beta_{2}}\left(\xi_{1}, \xi_{2}\right)=$ $f_{\beta_{1}}\left(\xi_{1}\right) f_{\beta_{2}}\left(\xi_{2}\right)$, where $\left\{f_{\beta_{i}}(\cdot)\right\}_{i=1}^{2}$ are the PDFs of univariate Nakagami- $m$ RVs [24, Eq. (2.20)]:

$$
\left\{f_{\beta_{i}}\left(\xi_{i}\right)\right\}_{i=1}^{2}=\tilde{A}_{i} \xi_{i}^{\tilde{C}_{i}} \exp \left(-\tilde{B}_{i} \xi_{i}^{2}\right)
$$

and we have defined $\tilde{A}_{i}=\left(2 m_{i}^{m_{i}}\right) /\left(\Omega_{i}^{m_{i}} \Gamma\left(m_{i}\right)\right), \tilde{B}_{i}=$ $m_{i} / \Omega_{i}$, and $\tilde{C}_{i}=2 m_{i}-1$.

By plugging (18) into (17), the integrals $\left\{P_{k}^{>}(p)\right\}_{p=1}^{2}$ and $\left\{P_{k}^{<}(p)\right\}_{p=1}^{2}$ can be computed in a single-integral closedform as summarized in Appendix A.

\section{E. Correlated Fading}

Let us analyze the scenario with correlated fading envelopes when $m_{1}=m_{2}=m$. In this case, the channel envelopes, $\left\{\beta_{i}\right\}_{i=1}^{2}$, are distributed according to a bivariate Nakagami- $m$ distribution with joint PDF, $f_{\beta_{1}, \beta_{2}}(\cdot, \cdot)$, as follows [24, Eq. (6.1)]:

$$
\begin{aligned}
f_{\beta_{1}, \beta_{2}}\left(\xi_{1}, \xi_{2}\right) & =A \exp \left(-B_{1} \xi_{1}^{2}\right) \exp \left(-B_{2} \xi_{2}^{2}\right) \\
& \times \xi_{1}^{m} \xi_{2}^{m} I_{m-1}\left(C \xi_{1} \xi_{2}\right)
\end{aligned}
$$

where we have defined:

$$
\left\{\begin{array}{l}
A=\frac{4 m^{m+1}}{\Gamma(m) \Omega_{1} \Omega_{2}\left(1-\rho_{\beta_{1}^{2} \beta_{2}^{2}}\right)\left(\sqrt{\Omega_{1} \Omega_{2} \rho_{\beta_{1}^{2} \beta_{2}^{2}}}\right)^{m-1}} \\
\left\{B_{i}\right\}_{i=1}^{2}=\frac{m}{\Omega_{i}\left(1-\rho_{\beta_{1}^{2} \beta_{2}^{2}}\right)} \\
C=\frac{2 m \sqrt{\rho_{\beta_{1}^{2} \beta_{2}^{2}}}}{\sqrt{\Omega_{1} \Omega_{2}}\left(1-\rho_{\beta_{1}^{2} \beta_{2}^{2}}\right)}
\end{array}\right.
$$

The ABEP for correlated fading can be obtained by exploiting the results already developed in Section IV-D. In particular, by using the infinite series representation of the $I_{m-1}(\cdot)$ Bessel function [20, Eq. (9.6.10)], (19) can be rewritten as shown in (21) on top of the next page, with $\Phi(\cdot, \cdot ; \cdot)$ 


$$
f_{\beta_{1} \beta_{2}}\left(\xi_{1}, \xi_{2}\right)=\sum_{q=0}^{+\infty}\left[\frac{4 m^{2 m+2 q}}{(q !) \Gamma(m) \Gamma(m+q)} \frac{\rho_{\beta_{1}^{2} \beta_{2}^{2}}^{q}}{\left(1-\rho_{\beta_{1}^{2} \beta_{2}^{2}}\right)^{m+2 q}} \frac{1}{\Omega_{1}^{m+q} \Omega_{2}^{m+q}} \Phi\left(\xi_{1}, \xi_{2} ; q\right)\right]
$$

$$
\left\{\begin{array}{l}
\left\{P_{k}^{>}(p)\right\}_{p=1}^{2}=\sum_{q=0}^{+\infty}\left[\frac{4 m^{2 m+2 q}}{(q !) \Gamma(m) \Gamma(m+q)} \frac{\rho_{\beta_{1}^{2} \beta_{2}^{2}}^{q}}{\left(1-\rho_{\beta_{1}^{2} \beta_{2}^{2}}\right)^{m+2 q}} \frac{1}{\Omega_{1}^{m+q} \Omega_{2}^{m+q}} P_{k}^{>}(p ; q)\right] \\
\left\{P_{k}^{<}(p)\right\}_{p=1}^{2}=\sum_{q=0}^{+\infty}\left[\frac{4 m^{2 m+2 q}}{(q !) \Gamma(m) \Gamma(m+q)} \frac{\rho_{\beta_{1}^{2} \beta_{2}^{2}}^{q}}{\left(1-\rho_{\beta_{1}^{2} \beta_{2}^{2}}\right)^{m+2 q}} \frac{1}{\Omega_{1}^{m+q} \Omega_{2}^{m+q}} P_{k}^{<}(p ; q)\right.
\end{array}\right]
$$

$$
f_{\beta_{1} \beta_{2}}\left(\xi_{1}, \xi_{2}\right)=f_{\beta_{1}}\left(\xi_{1}\right) f_{\beta_{2}}\left(\xi_{2}\right) \sum_{r=0}^{+\infty} \sum_{k=0}^{r} F(r, k) L_{r+k}^{\left(m_{1}-1\right)}\left(\frac{m_{1}}{\Omega_{1}} \xi_{1}^{2}\right) L_{r+k}^{\left(m_{2}-1\right)}\left(\frac{m_{2}}{\Omega_{2}} \xi_{2}^{2}\right)
$$

$$
f_{\beta_{1} \beta_{2}}\left(\xi_{1}, \xi_{2}\right)=\sum_{r=0}^{+\infty} \sum_{k=0}^{r} \sum_{t_{1}=0}^{r+k} \sum_{t_{2}=0}^{r+k}\left[G\left(r, k, t_{1}, t_{2}\right) \Psi\left(\xi_{1}, \xi_{2} ; r, k, t_{1}, t_{2}\right)\right]
$$

being defined as follows:

$$
\begin{aligned}
\Phi\left(\xi_{1}, \xi_{2} ; q\right) & =\xi_{1}^{2 m+2 q-1} \xi_{2}^{2 m+2 q-1} \\
& \times \exp \left[-\frac{m}{\Omega_{1}\left(1-\rho_{\beta_{1}^{2} \beta_{2}^{2}}\right)} \xi_{1}^{2}\right] \\
& \times \exp \left[-\frac{m}{\Omega_{2}\left(1-\rho_{\beta_{1}^{2} \beta_{2}^{2}}\right)} \xi_{2}^{2}\right]
\end{aligned}
$$

We can notice that $(22)$ is written in a very convenient form which resembles, for an adequate choice of the parameters, to the product of two PDFs in (18). In particular, $\Phi\left(\xi_{1}, \xi_{2} ; q\right)=$ $f_{\beta_{1}}\left(\xi_{1} ; q\right) f_{\beta_{2}}\left(\xi_{2} ; q\right)$ with:

$$
\left\{\begin{array}{l}
\left\{\tilde{A}_{i}\right\}_{i=1}^{2}=1 \\
\left\{\tilde{B}_{i}\right\}_{i=1}^{2}=m\left[\Omega_{i}\left(1-\rho_{\beta_{1}^{2} \beta_{2}^{2}}\right)\right]^{-1} \\
\left\{\tilde{C}_{i}(q)\right\}_{i=1}^{2}=2 m+2 q-1
\end{array}\right.
$$

Thus, the formula for the ABEP in (16) can still be used with the definitions summarized in (24) on top of this page, where $\left\{P_{k}^{>}(p ; q)\right\}_{p=1}^{2}$ and $\left\{P_{k}^{<}(p ; q)\right\}_{p=1}^{2}$ can be obtained from (35)-(40) in Appendix A by taking into account (23).

As a final remark, we observe that, although the final result in (24) requires an infinite series to compute the ABEP, this series is absolutely convergent, and converges rapidly thanks to the factorial term and the Gamma function in its denominator, i.e., only a few terms are required to obtain a good accuracy.

\section{F. Correlated Fading With Arbitrary Correlation and Fading Parameters}

Let us now analyze the very general setup with arbitrary correlation and fading parameters. A general PDF for the bivariate Nakagami- $m$ distribution has been recently introduced in [25], where the limitations of previous models have been overcome. In particular, it has been shown that the most general expression for the PDF of a bivariate Nakagami- $m$ distribution can be written as shown in (25) on top of this page [25, Eq. (12)], where (with $m_{2} \geq m_{1}$ ):

$$
\begin{aligned}
F(r, k) & =\frac{\left(m_{1} / 2\right)_{r}}{r !}\left(\begin{array}{l}
r \\
k
\end{array}\right)(-1)^{k} \bar{\delta}^{2 k} \tilde{\delta}^{r-k} \\
& \times \frac{(r+k) !}{\left(m_{1} / 2\right)_{r+k}} \frac{(r+k) !}{\left(m_{2} / 2\right)_{r+k}}
\end{aligned}
$$

and $\left\{f_{\beta_{i}}(\cdot)\right\}_{i=1}^{2}$ are defined in (18), $\bar{\delta}=\delta_{1} \delta_{2}-\delta_{3} \delta_{4}$, and $\tilde{\delta}=\delta_{1}^{2}+\delta_{2}^{2}+\delta_{3}^{2}+\delta_{4}^{2}$. In particular, $\left\{\delta_{h}\right\}_{h=1}^{4}$ are the correlation coefficients between the pairs of Rayleigh RVs composing the two Nakagami- $m$ RVs with PDF shown in (25) [25, Fig. 1 and Eq. (2)]. Moreover, the power correlation coefficient is given by $\rho_{\beta_{1}^{2} \beta_{2}^{2}}=0.5 \tilde{\delta} \sqrt{m_{1} / m_{2}}$.

By exploiting the identity [25, Eq. (9)], an equivalent form of the PDF in (25) is shown in (27) on top of this page, where:

$$
\begin{aligned}
\Psi\left(\xi_{1}, \xi_{2} ; r, k, t_{1}, t_{2}\right) & =\tilde{A}_{1} \tilde{A}_{2} \xi_{1}^{2 m_{1}-1+2 t_{1}} \xi_{2}^{2 m_{2}-1+2 t_{2}} \\
& \times \exp \left[-\tilde{B}_{1} \xi_{1}^{2}\right] \exp \left[-\tilde{B}_{2} \xi_{2}^{2}\right] \\
G\left(r, k, t_{1}, t_{2}\right)= & \tilde{B}_{1}^{t_{1}} \tilde{B}_{2}^{t_{2}} F(r, k) \\
& \times \frac{(-1)^{t_{1}} \Gamma\left(m_{1}+r+k\right)}{t_{1} !\left(r+k-t_{1}\right) ! \Gamma\left(m_{1}+t_{1}\right)} \\
& \times \frac{(-1)^{t_{2}} \Gamma\left(m_{2}+r+k\right)}{t_{2} !\left(r+k-t_{2}\right) ! \Gamma\left(m_{2}+t_{2}\right)}
\end{aligned}
$$

By looking at (27), we note that also in this case the PDF, $f_{\beta_{1} \beta_{2}}(\cdot, \cdot)$, is written in a form convenient to exploit the development already described for independent fading. In particular, by using arguments similar to Section IV-E, the ABEP in (16) can still be used with these definitions: 


$$
\left\{\begin{aligned}
\left\{P_{k}^{>}(p)\right\}_{p=1}^{2} & =\sum_{r=0}^{+\infty} \sum_{k=0}^{r} \sum_{t_{1}=0}^{r+k} \sum_{t_{2}=0}^{r+k}\left[G\left(r, k, t_{1}, t_{2}\right) P_{k}^{>}(p ; q)\right] \\
\left\{P_{k}^{<}(p)\right\}_{p=1}^{2} & =\sum_{r=0}^{+\infty} \sum_{k=0}^{r} \sum_{t_{1}=0}^{r+k} \sum_{t_{2}=0}^{r+k}\left[G\left(r, k, t_{1}, t_{2}\right) P_{k}^{<}(p ; q)\right]
\end{aligned}\right.
$$

where $\left\{P_{k}^{>}(p ; q)\right\}_{p=1}^{2},\left\{P_{k}^{<}(p ; q)\right\}_{p=1}^{2}$ can be obtained from (35)-(40) in Appendix A with $\left\{\tilde{C}_{i}\left(t_{i}\right)\right\}_{i=1}^{2}=2 m_{i}-1+2 t_{i}$.

We observe that, although the PDF in (25) is the most general expression of the bivariate Nakagami- $m$ distribution, it is written in a convenient form suitable for further analysis, which has lead to (30). Let us also emphasize that, even though, as mentioned in [25], (25) includes as special cases (18) and (19), we have decided to analyze all the cases in order to have simpler final expressions for the spacial cases analyzed in Section IV-D and Section IV-E. Finally, we also observe that all the results above are useful for any values of the fading parameters $\left\{m_{i}\right\}_{i=1}^{2}$, which are not restricted to be integer or half-integer values [25].

\section{G. A Simple Approximation to Reduce the Computational Complexity}

The ABEP in (16) requires the numerical computation of the finite-limit integrals summarized in Appendix A, which, in general, does not require any significant effort. However, we also notice that for correlated fading the number of integrals to be computed numerically could be non-negligible due to the series expressions of the PDFs in (21) and (27). With the aim to reduce the computational complexity of the framework, we propose in this sub-section a simple approximation which allows us to reduce the computational complexity of some integrals in Appendix A. In particular, the computation of integrals involving the Meijer-G function can be, sometimes, numerically time-consuming. So, we propose a simple approximation to circumvent this problem. We will validate in Section VI that the proposed approximation turns out to be very accurate, especially for high SNRs and for pragmatic values of the ABEP.

The reader can readily verify by direct inspection, and by following the procedure described in Appendix A, that the Meijer-G function arises from the computation of integrals that can be cast in the parametric form as follows:

$$
\mathrm{Z}=\int_{0}^{+\infty} x^{\nu} \exp \left(-\alpha x^{2}\right) \operatorname{erfc}(\beta x) d x
$$

with $\nu \geq 0, \alpha>0$, and $\beta>0$.

Instead of using the Mellin-Barnes theorem [21] to compute the integral in (31), we suggest to exploit the approximation in [31, Eq. (14)] for the erfc (.). In particular, (31) can be re-written as follows:

$$
\begin{aligned}
\mathrm{Z} & \cong \sum_{b=1}^{2}\left\{\omega_{b} \int_{0}^{+\infty} x^{\nu} \exp \left[-\left(\alpha+\eta_{b} \beta^{2}\right) x^{2}\right] d x\right\} \\
& =\sum_{b=1}^{2}\left[\frac{\omega_{b}}{2} \Gamma\left(\frac{\nu+1}{2}\right)\left(\alpha+\eta_{b} \beta^{2}\right)^{\frac{\nu+1}{2}}\right]
\end{aligned}
$$

where we have defined $\left\{\omega_{b}\right\}_{b=1}^{2}=\{1 / 6,1 / 2\},\left\{\eta_{b}\right\}_{b=1}^{2}=$ $\{1,4 / 3\}$, and the last identity is obtained by using [29, Eq. (3.462.1)].

By exploiting (32), the integrals in (36), (38), (40) in Appendix A simplify as summarized in (41)-(43) in Appendix $\mathrm{B}$, respectively.

\section{ABEP OVER NAKAGAMI- $m$ FADING ChanNels: THE $N_{t} \times 1$ MISO SETUP}

In Section IV, we have provided exact, even though expressed in the form of a single-integral to be computed numerically, expressions of the ABEP when the transmitter is equipped with two transmit-antennas. In this section, the frameworks are generalized to account for an arbitrary number of antennas at the transmitter. However, due to the evident complexity of analyzing the performance of the detector in Section III, even for the system setup with $N_{t}=2$, we do not attempt to compute the exact performance of it when $N_{t}>2$. On the contrary, we capitalize on two asymptoticallytight bounds that have been recently introduced in [12] for performance analysis of F-CSI-assisted detectors. In [12], it has been shown that these bounds yield a better accuracy than other bounds already available in the literature (see, e.g., [4, Eq. (4)]). In this paper, we analyze the tightness of them when a P-CSI-assisted detector is considered. For the convenience of the reader, these bounds are summarized in what follows. Further details about their derivation can be found in [12] and are not reproduced here for the sake of conciseness and to avoid redundancy. In Section VI, the tightness of both bounds will be validated via Monte Carlo simulations.

1) Symbol-based Union Bound: The first bound, which is called Symbol-based Union Bound (SUB), can be obtained by using typical methods for performance analysis of multi-level modulation schemes with optimum detection, as follows [12, Eq. (33)]:

$$
\begin{aligned}
\mathrm{ABEP} & \leq \mathrm{ABEP}^{\mathrm{SUB}} \\
& =\frac{1}{\left(N_{t}-1\right)} \sum_{i_{1}=1}^{N_{t}} \sum_{i_{2}=i_{1}+1}^{N_{t}} \mathrm{PEP}\left(\mathrm{TX}_{i_{1}} \rightarrow \mathrm{TX}_{i_{2}}\right)
\end{aligned}
$$

where PEP $\left(\mathrm{TX}_{i_{1}} \rightarrow \mathrm{TX}_{i_{2}}\right)$ denotes the Pairwise Error Probability (PEP) of the transmit-antennas $\mathrm{TX}_{i_{1}}$ and $\mathrm{TX}_{i_{2}}$ with $i_{1}, i_{2}=1,2, \ldots, N_{t}$, i.e., the probability of detecting $\operatorname{TX}_{i_{2}}$ when, instead, $\mathrm{TX}_{i_{1}}$ is actually transmitting. In particular, $\operatorname{PEP}\left(\mathrm{TX}_{i_{1}} \rightarrow \mathrm{TX}_{i_{2}}\right)$ is the ABEP of an equivalent $2 \times 1$ MISO system where only the transmit-antennas $\mathrm{TX}_{i_{1}}$ and $\mathrm{TX}_{i_{2}}$ can be activated for transmission. In other words, $\mathrm{PEP}\left(\mathrm{TX}_{i_{1}} \rightarrow \mathrm{TX}_{i_{2}}\right)$ in (33) is the ABEP computed in Section IV when $N_{t}=2$, i.e., (16).

2) Codeword-based Union Bound: The second bound, which is called Codeword-based Union Bound (CUB), can be obtained by using typical methods for performance analysis of Maximum Likelihood Sequence Estimation (MLSE) detectors as shown in (34) on top of the next page [12, Eq. (34)], where $N_{b}\left(i_{1}, i_{2}\right)$ is the number of information bit errors committed by choosing $\mathrm{TX}_{i_{2}}$ instead of $\mathrm{TX}_{i_{1}}$ as transmit-antenna. 


$$
\mathrm{ABEP} \leq \mathrm{ABEP}{ }^{\mathrm{CUB}}=\frac{2}{N_{t} \log _{2}\left(N_{t}\right)} \sum_{i_{1}=1}^{N_{t}} \sum_{i_{2}=i_{1}+1}^{N_{t}} N\left(i_{1}, i_{2}\right) \operatorname{PEP}\left(\mathrm{TX}_{i_{1}} \rightarrow \mathrm{TX}_{i_{2}}\right)
$$

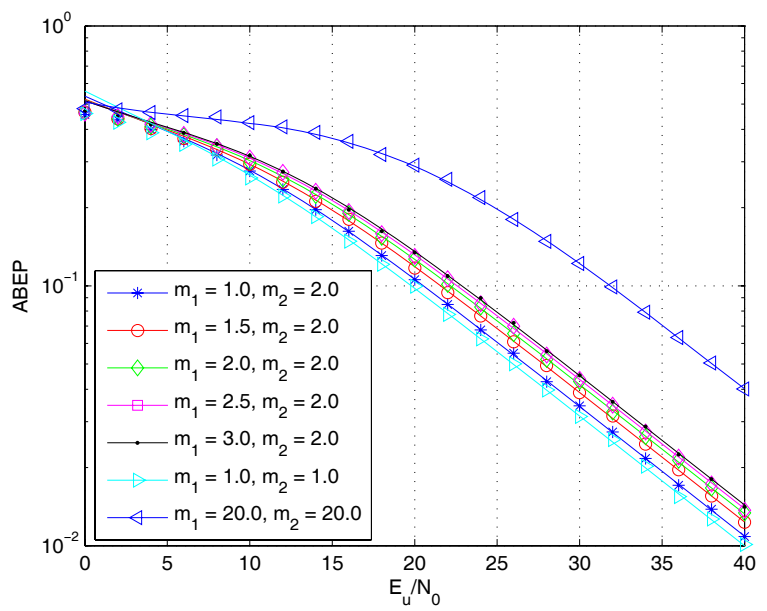

Fig. 1. Comparison between Monte Carlo simulation (markers) and analytical model (solid lines). $2 \times 1$ MISO system. Uncorrelated fading model in (18) (i.e., $\rho_{\beta_{1}^{2} \beta_{2}^{2}}=0$ ) with balanced power (i.e., $\Omega_{1}=\Omega_{2}=1$ ).

\section{NUMERicAl AND Simulation RESUltS}

In this section, we provide some numerical results with a threefold objective: i) to validate the accuracy of the analytical frameworks developed in Section IV and Section V, ii) to analyze the performance of SSK modulation for different fading parameters, i.e., fading correlation, fading severity, and power imbalance among the wireless links (i.e., $\left\{\Omega_{i}\right\}_{i=1}^{N_{t}}$ are different), and iii) to compare the performance of F-CSIand P-CSI-assisted detectors and quantify the performance loss caused by the simpler receiver design with unknown phase reference. Since various fading conditions are analyzed, the system setup used to obtain the numerical examples is shown for each figure in its caption. Monte Carlo simulations are obtained by using the simulation framework proposed in $[32]^{6}$ and [25] to generate bivariate Nakagami- $m$ fading envelopes with the PDF shown in (19) and (25), respectively. On the other hand, when $N_{t}>2$ Monte Carlo simulations are obtained by using the simulation framework introduced in [33] to generate multivariate Nakagami- $m$ fading envelopes. Moreover, unless otherwise stated, the series in (21) is truncated to the first 15 and 10 terms for $N_{t}=4$ and $N_{t}=8$, respectively, while the series in (25) is truncated to the first 3 terms. Finally, the integrals in (35)-(40) are computed by using straightforward numerical integration techniques.

a) Uncorrelated Fading: In Fig. 1 and Fig. 2, the scenario with uncorrelated fading for a balanced and an unbalanced setup is shown, respectively. By comparing the two figures, the following observations can be made. i) The proposed analytical model is very accurate and well overlaps with Monte Carlo simulations for various system settings. ii) The system performance improves for unbalanced fading

\footnotetext{
${ }^{6}$ Note that some typos in [32] have been adequately fixed and carefully taken into account to produce Monte Carlo simulation results [12, Appendix].
}

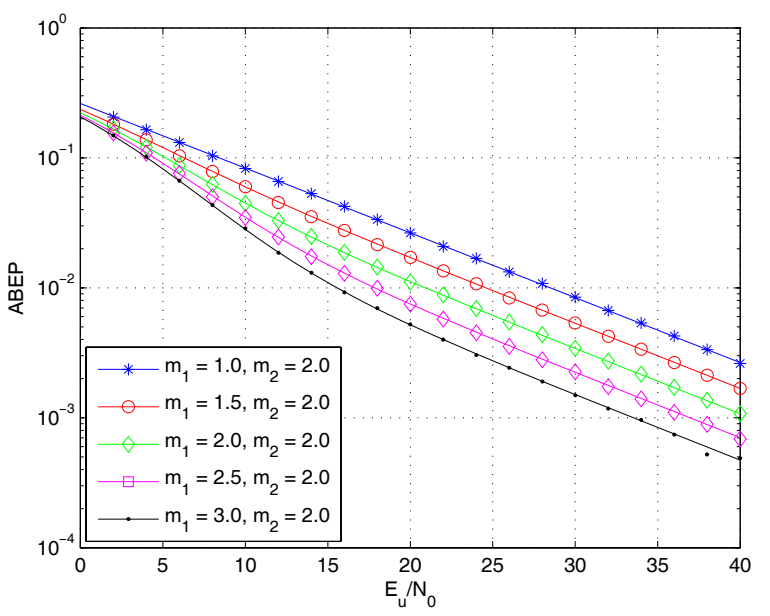

Fig. 2. Comparison between Monte Carlo simulation (markers) and analytical model (solid lines). $2 \times 1$ MISO system. Uncorrelated fading model in (18) (i.e., $\rho_{\beta_{1}^{2} \beta_{2}^{2}}=0$ ) with unbalanced power (i.e., $\Omega_{1}=10 \Omega_{2}$ ), and $\Omega_{2}=1$.

because the two links are more distinguishable from each other $^{7}$. This result can be readily understood by carefully looking at the ABEP in (15). As a matter of fact, all terms in (13) and (14) depend on the difference between the fading envelopes $\left\{\beta_{i}\right\}_{i=1}^{2}$, and the more the wireless links are unbalanced the more the addends in (13) and (14) are smaller. More specifically, when the wireless links are unbalanced both addends in (13) tend to zero, while the addends in (14) tend to cancel out in pairs, i.e., $P_{1}(\cdot, \cdot)$ with $P_{3}(\cdot, \cdot)$ and $P_{2}(\cdot, \cdot)$ with $P_{4}(\cdot, \cdot)$. This confirms that the proposed framework, besides being accurate, is also insightful about the behavior of the system. This trend confirms the findings already obtained in [12] for F-CSI-assisted detectors. iii) When a balanced fading scenario is considered, we observe an intriguing behavior of the ABEP, which was not observed for the F-CSI-assisted detector in [12]. In particular, in Fig. 1 we notice that the ABEP gets worse when the fading parameter $m_{1}$ increases. In other words, the performance gets worse when the Amount of Fading $\mathrm{AF}=1 / m_{1}$ [24, Eq. (2.4)] decreases. However, in general, it is expected that the ABEP gets better when the fading is less severe [24]. This apparently unexpected result can be readily understood by taking into account that, for balanced fading, the fading severity is the only responsible for making the wireless links more distinguishable from each

${ }^{7}$ With regard to the performance comparison between balanced and unbalanced setups, we emphasize here that the setups in Fig. 1 and Fig. 2 have a different average SNR per branch. As a consequence, the better performance achieved by the unbalanced setup with respect to the balanced setup is due, in part, to this latter aspect. To analyze the performance of two similar systems but with the same average SNR per branch in the balanced and unbalanced configurations, the reader is kindly requested to consult the comments in [12, Sec. 5]. A similar comment applies to several other curves shown in this paper if the reader is interested in comparing system setups with the same average SNR per branch. Finally, we emphasize here that power imbalance might arise in several cases and for many different reasons [12]. 


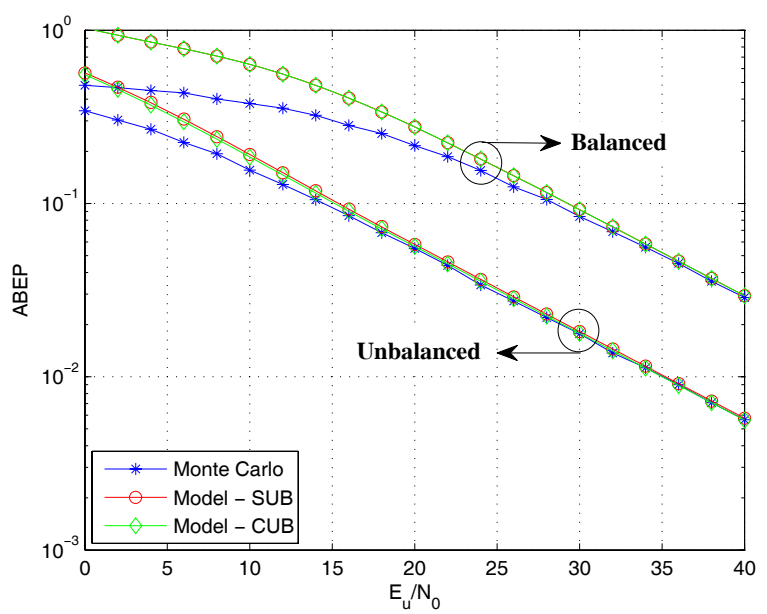

Fig. 3. Comparison between Monte Carlo simulation and analytical model. $4 \times 1$ MISO system. Uncorrelated fading model in (18) (i.e., $\left\{\rho_{\beta_{i}^{2} \beta_{j}^{2}}\right\}_{i \neq j=1}^{4}=0$ ) with balanced (i.e., $\left.\left\{\Omega_{i}\right\}_{i=1}^{4}=1\right)$ and unbalanced (i.e., $\Omega_{1}=1,\left\{\Omega_{i}\right\}_{i=2}^{4}=4(i-1)$ ) power, and $\left\{m_{i}\right\}_{i=1}^{4}=2.5$.

other: the more severe the fading is (higher AF), the more the links can be distinguished from each other. This behavior is not so evident for F-CSI detectors, which, for the same system setup, offered similar ABEP for various fading severities [12]. iv) On the other hand, for unbalanced fading we notice that the system behavior is the opposite: the ABEP gets better when the AF decreases. Moreover, the performance difference is non-negligible (greater than $10 \mathrm{~dB}$ in some cases). This behavior has already been observed for F-CSI detectors [12]. The reason is simple: when the wireless links are unbalanced, the fading could reduce the average gap between them due to deep fade fluctuations. As a consequence, less severe fading conditions are unlikely to offset the average power gap between the wireless links, thus yielding, on average, better error performance.

In Fig. 3, we show the ABEP when $N_{t}=4$ for both balanced and unbalanced system setups. We observe that both bounds summarized in Section $\mathrm{V}$ are very accurate and asymptotically tight also for P-CSI-assisted detectors. Moreover, we notice that: i) the system setup with unbalanced fading offers better performance than the balanced case, and ii) by comparing Fig. 1 and Fig. 2 with Fig. 3 we observe that the detector offers worse performance when the number of antennas at the transmitter increases. This trend is similar to that already obtained in [4] and [12] for the F-CSI-assisted detector.

b) Correlated Fading in (25): In Figs. 4-6, the scenario with correlated fading for a low and high fading severity, and balanced and unbalanced fading is shown. By carefully analyzing the figures, the following observations can be made. i) Also in this case, the proposed analytical model is very accurate and well overlaps with Monte Carlo simulations for various system settings. Moreover, we notice that the approximation introduced in Section IV-G is very accurate, especially in the high SNR region. It can be efficiently used for a simple performance analysis by still retaining a good accuracy. ii) Similar to the uncorrelated scenario, SSK modulation offers

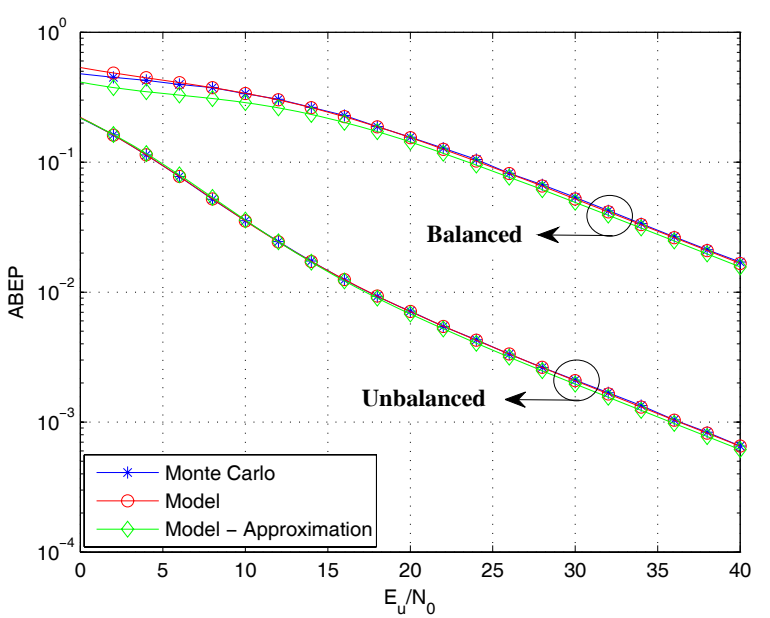

Fig. 4. Comparison between Monte Carlo simulation and analytical model. $2 \times 1$ MISO system. Correlated fading model in (25) with $\delta_{1}=\delta_{2}=\delta_{3}=$ 0.45 and $\delta_{4}=-0.45$. Balanced (i.e., $\Omega_{1}=\Omega_{2}=1$ ) and unbalanced (i.e., $\Omega_{1}=10 \Omega_{2}$ ) power with $m_{1}=m_{2}=2.0$.

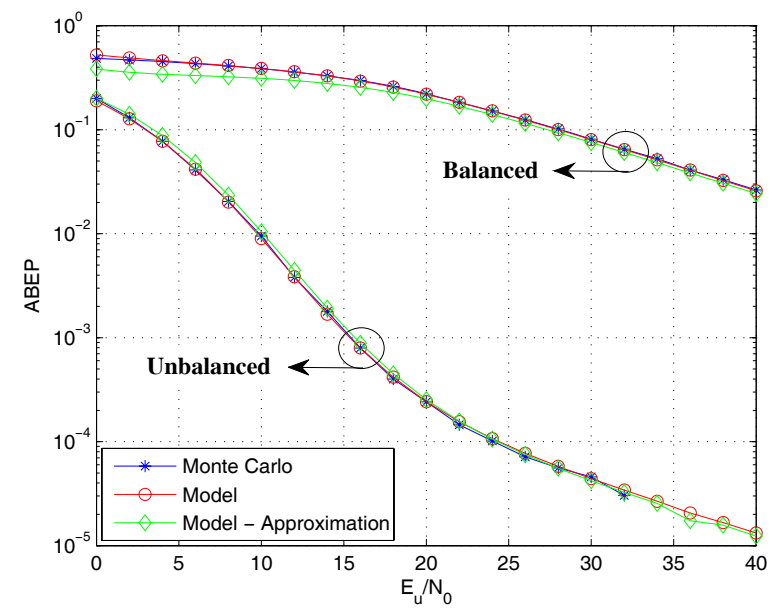

Fig. 5. Comparison between Monte Carlo simulation and analytical model. $2 \times 1$ MISO system. Correlated fading model in (25) with $\delta_{1}=\delta_{2}=\delta_{3}=$ 0.45 and $\delta_{4}=-0.45$. Balanced (i.e., $\Omega_{1}=\Omega_{2}=1$ ) and unbalanced (i.e., $\Omega_{1}=10 \Omega_{2}$ ) power with $m_{1}=m_{2}=5.0$.

better performance for unbalanced fading conditions, since, in this case, the wireless links are more distinguishable from each other. Moreover, for unbalanced fading the ABEP gets better when $\left\{m_{i}\right\}_{i=1}^{2}$ increase, while for balanced fading it gets worse. This trend is similar to the system setup with uncorrelated fading. However, we can observe a huge (and a priori unpredictable) performance difference (greater than $30 \mathrm{~dB}$ ) between balanced and unbalanced settings in Fig. 5 . iii) When comparing the performance of uncorrelated and uncorrelated fading for the same setting (e.g., Fig. 1, Fig. 2 and Fig. 4 when $m_{1}=m_{2}=2.0$ ), we notice a different behavior of the system with respect to channel correlation as far as balanced and unbalanced fading are concerned. In particular, in the presence of channel correlation the ABEP gets slightly worse when the wireless links are balanced, while it gets better when the wireless links are unbalanced. This apparently unexpected result has already been observed in [12] for the 


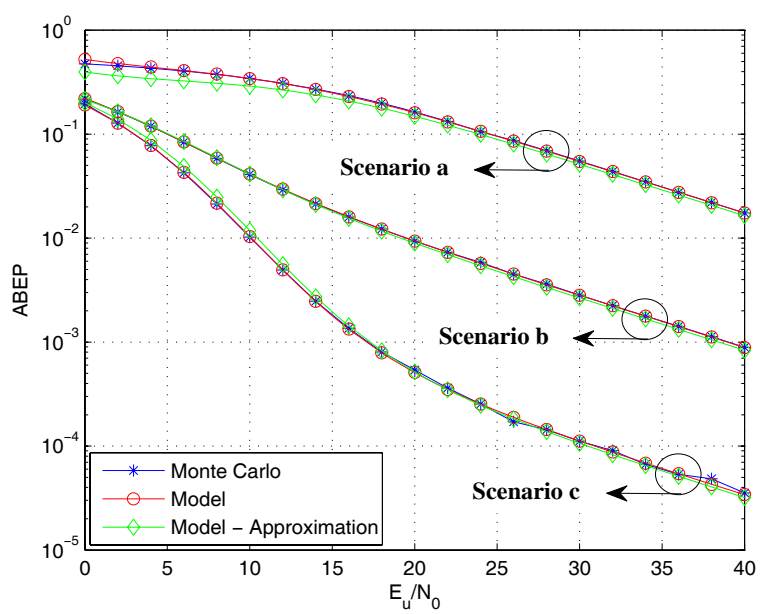

Fig. 6. Comparison between Monte Carlo simulation and analytical model. $2 \times 1$ MISO system. Correlated fading model in (25) with $\delta_{1}=\delta_{2}=\delta_{3}=$ 0.45 and $\delta_{4}=-0.45$. Scenario a: $\Omega_{1}=\Omega_{2}=1, m_{1}=2.0$, and $m_{2}=5.0$ (the same curves are obtained if $\Omega_{1}=\Omega_{2}=1, m_{1}=5.0$, and $m_{2}=2.0$ ). Scenario b: $\Omega_{1}=10, \Omega_{2}=1, m_{1}=2.0$, and $m_{2}=5.0$. Scenario c: $\Omega_{1}=10, \Omega_{2}=1, m_{1}=5.0$, and $m_{2}=2.0$.

F-CSI-assisted detector and can be explained by still using the concept of AF: when the wireless links are unbalanced and correlated the fading fluctuations are less likely to offset the average power gap because they change jointly. On the contrary, when the wireless links are uncorrelated but still unbalanced, the links fade independently and the average power gap between them can be offset more often. iv) Finally, an interesting comment can be made by carefully observing Fig. 6, and, in particular, the curves related to Scenario $b$ and Scenario $c$. In both system setups the first wireless link has a greater power gain, but in Scenario $b$ the AF of the first link is greater than the $\mathrm{AF}$ of the second link. We can observe a significant performance difference, which highlights that besides the average power also the fading severity can remarkably alter the system performance. This result substantiates the possible adoption of opportunistic power allocation mechanisms for the optimization of the performance of SSK modulation over fading channels. In [12], it has been remarked that power imbalance between the wireless links can be created artificially by allowing each antenna to transmit a different average power. The comparison between Scenario $b$ and Scenario $c$ in Fig. 6 brings to our attention that we can obtain higher performance gains by increasing the transmission power of the wireless link having the smaller AF. Depending on the channel fading model, the performance gain can also be significant, as shown in Fig. 6. A recent example can also be found in [34].

c) Correlated Fading in (19): In Figs. 7-10, we analyze the system performance when the correlated fading model in (19) is considered. In particular, the ABEP for a number of antennas greater than two is shown. Besides the good agreement between analytical model and Monte Carlo simulation, the better performance for unbalanced settings, as well as the different behavior of balanced and unbalanced setups for different fading severities (i.e., the fading parameter $m$ ), we can also study the behavior of the system for different values of the correlation coefficient, i.e., $d_{0}$. In particular, among the

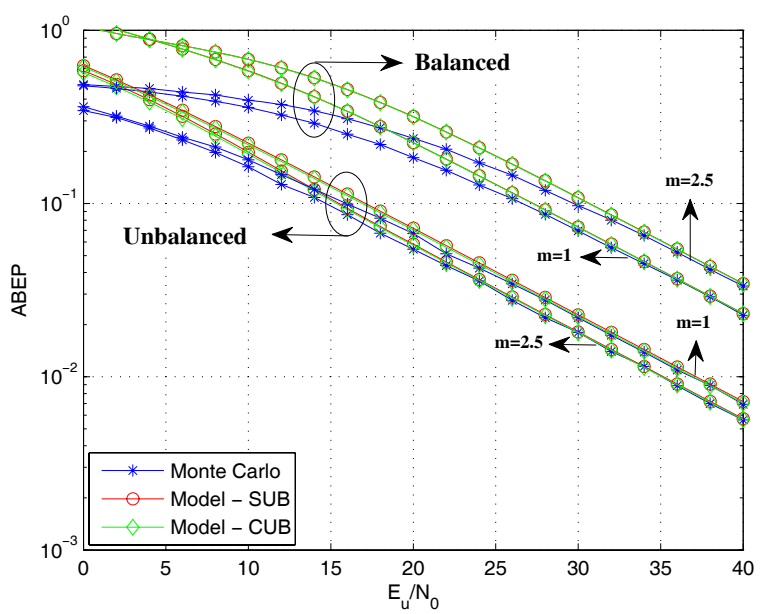

Fig. 7. Comparison between Monte Carlo simulation and analytical model. $4 \times 1$ MISO system. Correlated fading model in (19) (i.e., $\left\{\rho_{\beta_{i}^{2} \beta_{j}^{2}}\right\}_{i, j=1}^{4} \cong$ $\left.\left\{\rho_{\beta_{i} \beta_{j}}\right\}_{i, j=1}^{4}=\exp \left(-d_{0}|i-j|\right)\right)$ with balanced (i.e., $\left.\left\{\Omega_{i}\right\}_{i=1}^{4}=1\right)$ and unbalanced (i.e., $\Omega_{1}=1,\left\{\Omega_{i}\right\}_{i=2}^{4}=4(i-1)$ ) power, and $d_{0}=0.91$.

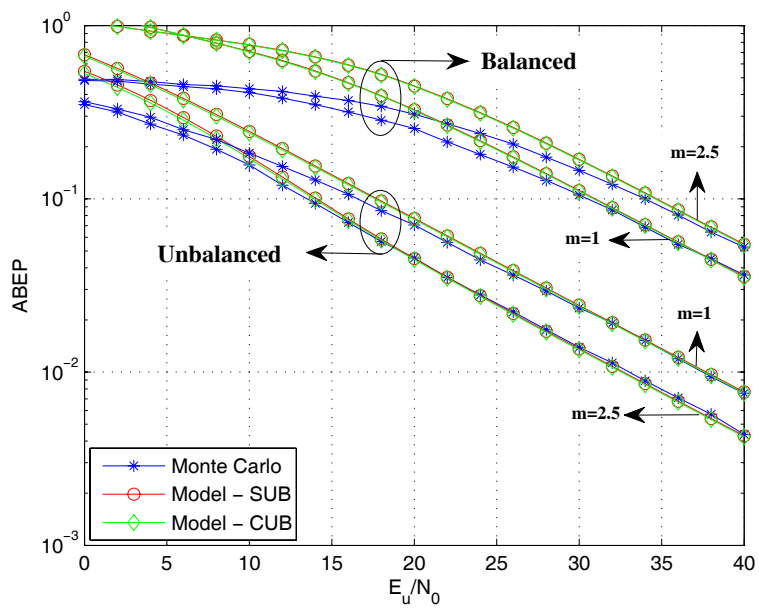

Fig. 8. Comparison between Monte Carlo simulation and analytical model. $4 \times 1$ MISO system. Correlated fading model in (19) (i.e., $\left\{\rho_{\beta_{i}^{2} \beta_{j}^{2}}\right\}_{i, j=1}^{4} \cong$ $\left.\left\{\rho_{\beta_{i} \beta_{j}}\right\}_{i, j=1}^{4}=\exp \left(-d_{0}|i-j|\right)\right)$ with balanced (i.e., $\left.\left\{\Omega_{i}\right\}_{i=1}^{4}=1\right)$ and unbalanced (i.e., $\Omega_{1}=1,\left\{\Omega_{i}\right\}_{i=2}^{4}=4(i-1)$ ) power, and $d_{0}=0.22$. The series in (21) is truncated to the first 30 terms for the balanced system setup.

$N_{t}$ antennas we have a correlation coefficient in the range $[0.0625,0.4025]$ and $[0.5169,0.8025]$ when $d_{0}=0.91$ and $d_{0}=0.22$, respectively. We observe that the ABEP gets worse for larger values of the correlation coefficient for the balanced system setup, while the ABEP gets better for the unbalanced system setup. This trend confirms the results already obtained for $N_{t}=2$. Moreover, we note in Fig. 7 and Fig. 8 that, for the unbalanced setup, the performance gap for different fading severities increases with the correlation coefficient. Furthermore, by comparing the ABEP for four and eight transmitantennas we notice that it gets worse when $N_{t}$ increases, as expected. Furthermore, a trend similar to previous figures can be remarked as far as the fading severity is concerned. Finally, 


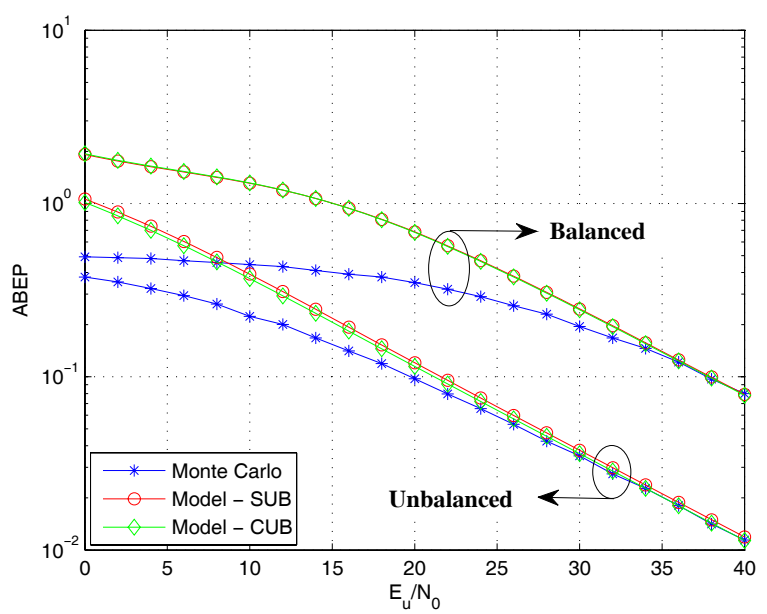

Fig. 9. Comparison between Monte Carlo simulation and analytical model. $8 \times 1$ MISO system. Correlated fading model in (19) (i.e., $\left\{\rho_{\beta_{i}^{2} \beta_{j}^{2}}\right\}_{i, j=1}^{8} \cong$ $\left.\left\{\rho_{\beta_{i} \beta_{j}}\right\}_{i, j=1}^{8}=\exp \left(-d_{0}|i-j|\right)\right)$ with balanced (i.e., $\left.\left\{\Omega_{i}\right\}_{i=1}^{8}=1\right)$ and unbalanced (i.e., $\Omega_{1}=1,\left\{\Omega_{i}\right\}_{i=2}^{8}=3(i-1)$ ) power, and $d_{0}=0.22$, $\left\{m_{i}\right\}_{i=1}^{8}=2.5$.

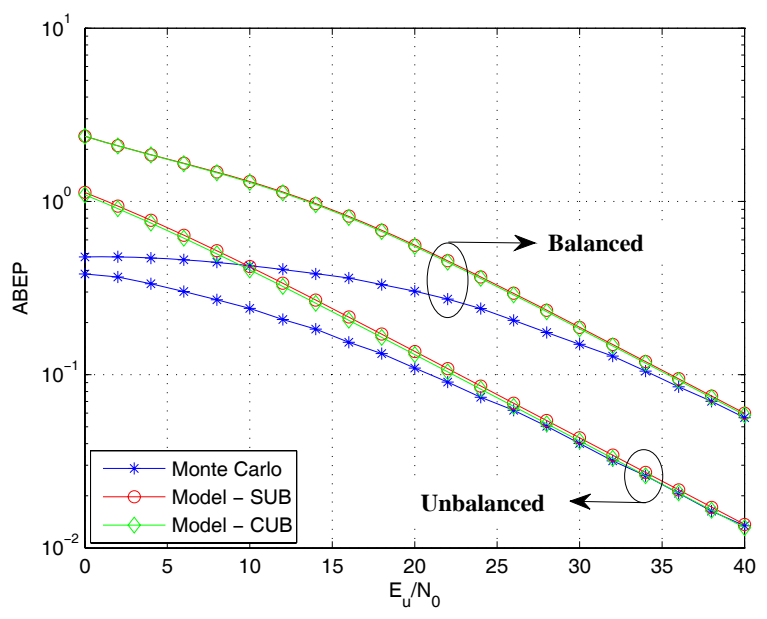

Fig. 10. Comparison between Monte Carlo simulation and analytical model. $8 \times 1$ MISO system. Correlated fading model in (19) (i.e., $\left\{\rho_{\beta_{i}^{2} \beta_{j}^{2}}\right\}_{i, j=1}^{8} \cong$ $\left.\left\{\rho_{\beta_{i} \beta_{j}}\right\}_{i, j=1}^{8}=\exp \left(-d_{0}|i-j|\right)\right)$ with balanced (i.e., $\left.\left\{\Omega_{i}\right\}_{i=1}^{8}=1\right)$ and unbalanced (i.e., $\Omega_{1}=1,\left\{\Omega_{i}\right\}_{i=2}^{8}=3(i-1)$ ) power, and $d_{0}=0.22$, $\left\{m_{i}\right\}_{i=1}^{8}=1$. The series in (21) is truncated to the first 20 terms for the balanced system setup.

we note that, as expected, the bounds are weak for low SNRs: this is typical of union bound methods [13]. However, the frameworks are very tight for $\mathrm{ABEP}<10^{-1}$, which is the setup where a pragmatic system is expected to operate.

d) Comparison with the F-CSI-Assisted Detector [12]: Finally, in Fig. 11 and Fig. 12, we compare the performance of P-CSI- and F-CSI-assisted detectors. In particular, the results of this latter detector have been obtained by using the analytical framework recently introduced in [12]. We can readily figure out that neglecting the channel phase information for a low-complexity implementation of the receiver (i.e., keeping at a low-complexity the channel estimator)

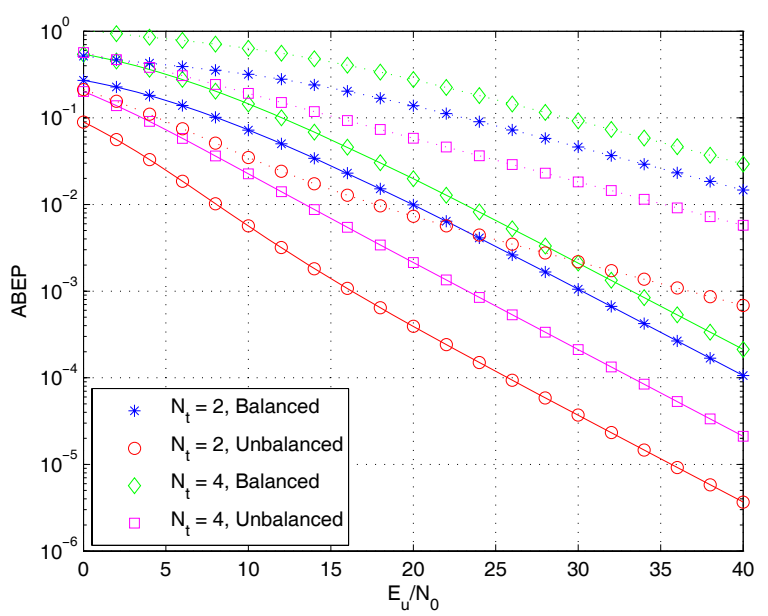

Fig. 11. Performance comparison (analytical model only) between PCSI (dotted lines with markers) and F-CSI (solid lines with markers) receivers [12]. $N_{t} \times 1$ MISO system. Uncorrelated fading model in (18) (i.e., $\left\{\rho_{\beta_{i}^{2} \beta_{j}^{2}}\right\}_{i \neq j=1}^{N_{t}}=0$ ). Balanced setup: $\left\{\Omega_{i}\right\}_{i=1}^{N_{t}}=1$. Unbalanced setup: $\Omega_{1}=10, \Omega_{2}=1$ if $N_{t}=2$, and $\Omega_{1}=1,\left\{\Omega_{i}\right\}_{i=2}^{4}=4(i-1)$ if $N_{t}=4 .\left\{m_{i}\right\}_{i=1}^{N_{t}}=2.5$.

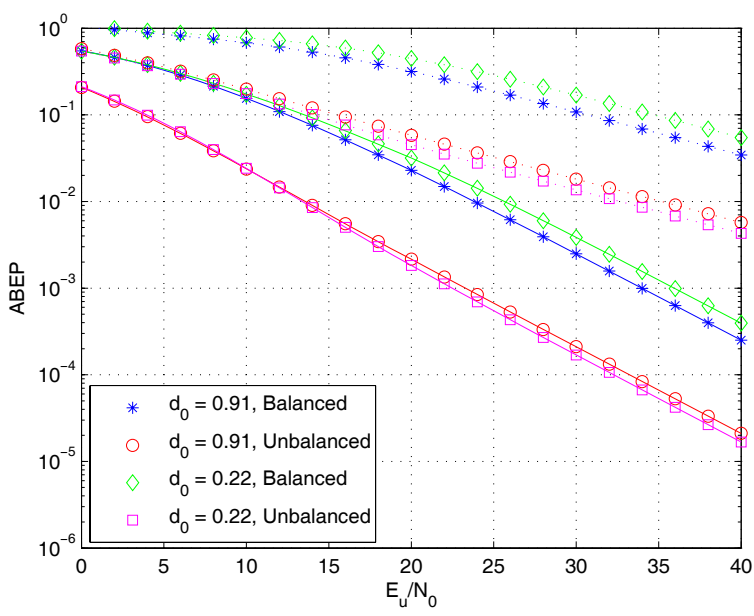

Fig. 12. Performance comparison (analytical model only) between PCSI (dotted lines with markers) and F-CSI (solid lines with markers) receivers [12]. $4 \times 1 \mathrm{MISO}$ system. Correlated fading model in (19) (i.e., $\left\{\rho_{\beta_{i}^{2} \beta_{j}^{2}}\right\}_{i, j=1}^{4} \cong\left\{\rho_{\beta_{i} \beta_{j}}\right\}_{i, j=1}^{4}=\exp \left(-d_{0}|i-j|\right)$ ). Balanced setup: $\left\{\Omega_{i}\right\}_{i=1}^{4}=1$. Unbalanced setup: $\Omega_{1}=1,\left\{\Omega_{i}\right\}_{i=2}^{4}=4(i-1)$. $\left\{m_{i}\right\}_{i=1}^{4}=2.5$.

may lead to a substantial performance loss with respect to a F-CSI system design. This result is in net contrast with ordinary modulation schemes in which the performance loss of a receiver with and without phase information is limited to a few dB. For example, BPSK and DBPSK receivers differ less than $1 \mathrm{~dB}$ over AWGN channels [13, Fig. 5.2.12], and approximately $3 \mathrm{~dB}$ over Rayleigh fading channels [13, Fig. 14.3.1]. A similar comment can be made for MIMO systems when comparing coherent and differential transmission schemes (see, e.g., [14]-[16]). This performance drop is due to the peculiar operations of SSK modulation with respect to ordinary modulation schemes: since it is the location-specific 
channel fingerprint which carries information, neglecting part of it (e.g., the channel phase in this paper) may lead to a significant performance degradation. As a consequence, the design and adoption of accurate channel estimation algorithms will play a fundamental role for the successful exploitation and deployment of SSK modulation in realistic environments. By looking at Fig. 11, we note that moving from 2 to 4 antennas the performance drop for the unbalanced setup is higher than that observed for the balanced setup. Both receivers show this trend. Finally, by looking at Fig. 12 we observe that channel correlation can either increase or decrease the ABEP of a few $\mathrm{dB}$ only: power imbalance has a more pronounced effect on the system performance than channel correlation.

\section{CONCLUSION}

In this paper, we have analyzed the performance of a novel detector for SSK modulation which does not require the knowledge of the channel phase. An accurate framework for analyzing its performance over correlated Nakagami- $m$ fading channels with arbitrary correlation and fading parameters has also been developed. Numerical results have validated the accuracy of the proposed analytical derivation, and shown that the system performance can change remarkably for different fading conditions. In particular, quite unexpected behaviors with respect to the fading severity have been evidenced. We have also verified that power imbalanced among the wireless links can significantly affect the system performance, even more than channel correlation. Finally, we have verified that the optimal detector with P-CSI can yield substantially worse performance than a F-CSI detector that can exploit the a priori knowledge of the channel phases. This is a result that is in net contrast with similar performance comparisons available for ordinary modulation schemes. All these trends can be well captured by the proposed analytical model. In summary, analysis and results obtained in this paper have highlighted that the adoption of accurate channel estimation algorithms will play a fundamental role for the successful exploitation of SSK modulation in realistic environments.

Ongoing research is now concerned with a comprehensive performance study and comparison between SSK modulation and other MIMO schemes, in order to fully understand the trade-off offered by SSK modulation in terms of performance, achievable throughput, and system complexity over realistic fading environments and for different levels of a priori channel knowledge at the transmitter and receiver.

\section{ACKNOWLEDGMENT}

We gratefully acknowledge support from the EPSRC (EP/G011788/1) for this work. Harald Haas acknowledges the Scottish Funding Council support of his position within the Edinburgh Research Partnership in Engineering and Mathematics between the University of Edinburgh and Heriot Watt University.

APPENDIX A

Computation of (17)

By plugging (18) into (17), $\left\{P_{k}^{>}(p)\right\}_{p=1}^{2}$ in (17) can be computed as shown in (35)-(40) on top of the next page.
In particular, the following procedure is adopted to get the results in (35)-(40). 1) Each integral is re-written by using the change of variable $x_{2}=\xi_{2} / \xi_{1}$. 2) The integral over the positive real axis is computed in closed-form by exploiting some notable integral results. In particular, i) (35), (37) and (39) are obtained by using [29, Eq. (3.462.1)], ii) (36) and (38) are obtained by using the identity erfc $(-x)=2-\operatorname{erfc}(x)$ $\forall x \geq 0$, as well as [29, Eq. (3.462.1)] and the Mellin-Barnes theorem in [21, Eq. (2.24.1.1)], and iii) (40) is computed by using [29, Eq. (3.462.1)] and the Mellin-Barnes theorem in [21, Eq. (2.24.1.1)]. All the integrals are conveniently written as a single finite-range integral, which can be readily computed with consolidated numerical integration methods. For example, Gauss-Legendre Quadrature Rules could be readily applied [30]. Finally, we note that, due to the symmetry of the problem at hand, the integrals $\left\{P_{k}^{<}(p)\right\}_{p=1}^{2}$ in (17) can be obtained from (35)-(40) by simply inverting the fading parameters of the wireless links, e.g., $\tilde{B}_{1}$ is replaced by $\tilde{B}_{2}$, $\tilde{C}_{2}$ by $\tilde{C}_{1}$, etc.

\section{APPENDIX B}

APPROXIMATED EXPRESSION FOR (36), (38), AND (40)

By using the approximation in (32), the integrals (36), (38) and (40) in Appendix A can be accurately computed as shown in (41)-(43) on top of the next page, respectively.

\section{REFERENCES}

[1] Y. Chau and S.-H. Yu, "Space modulation on wireless fading channels," in Proc. IEEE Veh. Technol. Conf.-Fall, Oct. 2001, vol. 3, pp. 16681671.

[2] H. Haas, E. Costa, and E. Schultz, "Increasing spectral efficiency by data multiplexing using antennas arrays," in Proc. IEEE Int. Symp. Personal, Indoor, Mobile Radio Commun., Sep. 2002, vol. 2, pp. 610-613.

[3] R. Y. Mesleh, H. Haas, S. Sinanovic, C. W. Ahn, and S. Yun, "Spatial modulation," IEEE Trans. Veh. Technol., vol. 57, no. 4, pp. 2228-2241, July 2008.

[4] J. Jeganathan, A. Ghrayeb, L. Szczecinski, and A. Ceron, "Space shift keying modulation for MIMO channels," IEEE Trans. Wireless Commun., vol. 8, no. 7, pp. 3692-3703, July 2009.

[5] L. Xiao, L. Greenstein, N. Mandayam, and W. Trappe, "Using the physical layer for wireless authentication in time-variant channels," IEEE Trans. Wireless Commun., vol. 7, no. 7, pp. 2571-2579, July 2008.

[6] J. Jeganathan, A. Ghrayeb, and L. Szczecinski, "Generalized space shift keying modulation for MIMO channels," in Proc. IEEE Int. Symp. Personal, Indoor, Mobile Radio Commun., Sep. 2008, pp. 1-5.

[7] R. Y. Mesleh, M. Di Renzo, H. Haas, and P. M. Grant, "Trellis coded spatial modulation," IEEE Trans. Wireless Commun., vol. 9, no. 7, pp. 2349-2361, July 2010.

[8] J. Jeganathan, A. Ghrayeb, and L. Szczecinski, "Spatial modulation: optimal detection and performance analysis," IEEE Commun. Lett., vol. 12, no. 8, pp. 545-547, Aug. 2008.

[9] P. Wolniansky, G. Foschini, G. Golden, and R. Valenzuela, "V-BLAST: an architecture for realizing very high data rates over the rich-scattering wireless channel," in Proc. IEEE Int. Symposium on Signals, Systems, and Electronics, Sep./Oct. 1998, pp. 295-300.

[10] S. M. Alamouti, "A simple transmit diversity technique for wireless communications," IEEE J. Sel. Areas Commun., vol. 16, no. 8, pp. 14511458, Oct. 1998.

[11] A. Alshamali and B. Quza, "Performance of spatial modulation in correlated and uncorrelated Nakagami fading channel," J. Commun., vol. 4, no. 3, pp. 170-174, Apr. 2009.

[12] M. Di Renzo and H. Haas, "A general framework for performance analysis of space shift keying (SSK) modulation for MISO correlated Nakagami- $m$ fading channels," IEEE Trans. Commun., vol. 58, no. 9, Sep. 2010.

[13] J. Proakis, Digital Communications, 4th edition. McGraw-Hill, 2000.

[14] V. Tarokh and H. Jafarkhani, "A differential detection scheme for transmit diversity," IEEE J. Sel. Areas Commun., vol. 18, no. 7, pp. 1169-1174, July 2000. 


$$
P_{1}^{>}(1)=\frac{\tilde{A}_{1} \tilde{A}_{2}}{2 \sqrt{\pi} \bar{\gamma}} \Gamma\left(\frac{\tilde{C}_{1}+\tilde{C}_{2}+1}{2}\right) \int_{0}^{1} \xi^{\tilde{C}_{2}}(1+\xi)^{-1 / 2}\left(\tilde{B}_{1}+\frac{\bar{\gamma}^{2}}{8}-\frac{\bar{\gamma}^{2}}{4} \xi+\tilde{B}_{2} \xi^{2}+\frac{\bar{\gamma}^{2}}{8} \xi^{2}\right)^{-\left(\frac{\tilde{C}_{1}+\tilde{C}_{2}+1}{2}\right)} d \xi
$$

$$
\begin{aligned}
& P_{2}^{>}(1)=\frac{\tilde{A}_{1} \tilde{A}_{2}}{\sqrt{2}} \Gamma\left(\frac{\tilde{C}_{1}+\tilde{C}_{2}+2}{2}\right) \int_{0}^{1} \xi^{\tilde{C}_{2}}(1+\xi)^{-1 / 2}\left(\tilde{B}_{1}+\tilde{B}_{2} \xi^{2}\right)^{-\left(\frac{\tilde{C}_{1}+\tilde{C}_{2}+2}{2}\right)} d \xi \\
& -\frac{\tilde{A}_{1} \tilde{A}_{2}}{2 \sqrt{2 \pi}} \int_{0}^{1} \xi^{\tilde{C}_{2}}(1+\xi)^{-1 / 2}\left(\tilde{B}_{1}+\tilde{B}_{2} \xi^{2}\right)^{-\left(\frac{\tilde{C}_{1}+\tilde{C}_{2}+2}{2}\right)} G_{2,2}^{2,1}\left(\frac{\bar{\gamma}^{2}(1-\xi)^{2}}{8\left(\tilde{B}_{1}+\tilde{B}_{2} \xi^{2}\right)} \mid \begin{array}{cc}
-\frac{\tilde{C}_{1}+\tilde{C}_{2}}{2} & 1 \\
0 & \frac{1}{2}
\end{array}\right) d \xi
\end{aligned}
$$

$$
P_{3}^{>}(1)=\frac{\tilde{A}_{1} \tilde{A}_{2}}{2 \sqrt{\pi} \bar{\gamma}} \Gamma\left(\frac{\tilde{C}_{1}+\tilde{C}_{2}+1}{2}\right) \int_{0}^{1} \xi^{\tilde{C}_{2}}(1+\xi)^{-1 / 2}\left(\tilde{B}_{1}+\frac{\bar{\gamma}^{2}}{2}+\tilde{B}_{2} \xi^{2}\right)^{-\left(\frac{\tilde{C}_{1}+\tilde{C}_{2}+1}{2}\right)} d \xi
$$

$$
\begin{aligned}
& P_{4}^{>}(1)=\frac{\tilde{A}_{1} \tilde{A}_{2}}{\sqrt{2}} \Gamma\left(\frac{\tilde{C}_{1}+\tilde{C}_{2}+2}{2}\right) \int_{0}^{1} \xi^{\tilde{C}_{2}}(1+\xi)^{-1 / 2}\left(\tilde{B}_{1}+\tilde{B}_{2} \xi^{2}\right)^{-\left(\frac{\tilde{C}_{1}+\tilde{C}_{2}+2}{2}\right)} d \xi \\
& -\frac{\tilde{A}_{1} \tilde{A}_{2}}{2 \sqrt{2 \pi}} \int_{0}^{1} \xi^{\tilde{C}_{2}}(1+\xi)^{-1 / 2}\left(\tilde{B}_{1}+\tilde{B}_{2} \xi^{2}\right)^{-\left(\frac{\tilde{C}_{1}+\tilde{C}_{2}+2}{2}\right)} G_{2,2}^{2,1}\left(\frac{\bar{\gamma}^{2}}{2\left(\tilde{B}_{1}+\tilde{B}_{2} \xi^{2}\right)} \mid \begin{array}{cc}
-\frac{\tilde{C}_{1}+\tilde{C}_{2}}{2} & 1 \\
0 & \frac{1}{2}
\end{array}\right) d \xi
\end{aligned}
$$

$$
P_{1}^{>}(2)=\frac{\tilde{A}_{1} \tilde{A}_{2}}{2 \sqrt{\pi} \bar{\gamma}} \Gamma\left(\frac{\tilde{C}_{1}+\tilde{C}_{2}+1}{2}\right) \int_{0}^{1} \xi^{\tilde{C}_{2}-1 / 2}(1+\xi)^{-1 / 2}\left(\tilde{B}_{1}+\frac{\bar{\gamma}^{2}}{8}-\frac{\bar{\gamma}^{2}}{4} \xi+\tilde{B}_{2} \xi^{2}+\frac{\bar{\gamma}^{2}}{8} \xi^{2}\right)^{-\left(\frac{\tilde{C}_{1}+\tilde{C}_{2}+1}{2}\right)} d \xi
$$

$$
P_{2}^{>}(2)=\frac{\tilde{A}_{1} \tilde{A}_{2}}{2 \sqrt{2 \pi}} \int_{0}^{1} \xi^{\tilde{C}_{2}+1 / 2}(1+\xi)^{-1 / 2}\left(\tilde{B}_{1}+\tilde{B}_{2} \xi^{2}\right)^{-\left(\frac{\tilde{C}_{1}+\tilde{C}_{2}+2}{2}\right)} G_{2,2}^{2,1}\left(\frac{\bar{\gamma}^{2}(1-\xi)^{2}}{8\left(\tilde{B}_{1}+\tilde{B}_{2} \xi^{2}\right)} \mid \begin{array}{cc}
-\frac{\tilde{C}_{1}+\tilde{C}_{2}}{2} & 1 \\
0 & \frac{1}{2}
\end{array}\right) d \xi
$$

$$
\begin{aligned}
P_{2}^{>}(1) & \cong \frac{\tilde{A}_{1} \tilde{A}_{2}}{\sqrt{2}} \Gamma\left(\frac{\tilde{C}_{1}+\tilde{C}_{2}+2}{2}\right) \int_{0}^{1} \xi^{\tilde{C}_{2}}(1+\xi)^{-1 / 2}\left(\tilde{B}_{1}+\tilde{B}_{2} \xi^{2}\right)^{-\left(\frac{\tilde{C}_{1}+\tilde{C}_{2}+2}{2}\right)} d \xi \\
& -\frac{\tilde{A}_{1} \tilde{A}_{2}}{2 \sqrt{2}} \Gamma\left(\frac{\tilde{C}_{1}+\tilde{C}_{2}+2}{2}\right) \sum_{b=1}^{2}\left[\omega_{b} \int_{0}^{1} \xi^{\tilde{C}_{2}}(1+\xi)^{-1 / 2}\left(\tilde{B}_{1}+\eta_{b} \frac{\bar{\gamma}^{2}(1-\xi)^{2}}{8}+\tilde{B}_{2} \xi^{2}\right)^{-\left(\frac{\tilde{C}_{1}+\tilde{C}_{2}+2}{2}\right)} d \xi\right]
\end{aligned}
$$

$$
\begin{aligned}
P_{4}^{>}(1) & \cong \frac{\tilde{A}_{1} \tilde{A}_{2}}{\sqrt{2}} \Gamma\left(\frac{\tilde{C}_{1}+\tilde{C}_{2}+2}{2}\right) \int_{0}^{1} \xi^{\tilde{C}_{2}}(1+\xi)^{-1 / 2}\left(\tilde{B}_{1}+\tilde{B}_{2} \xi^{2}\right)^{-\left(\frac{\tilde{C}_{1}+\tilde{C}_{2}+2}{2}\right)} d \xi \\
& -\frac{\tilde{A}_{1} \tilde{A}_{2}}{2 \sqrt{2}} \Gamma\left(\frac{\tilde{C}_{1}+\tilde{C}_{2}+2}{2}\right) \sum_{b=1}^{2}\left[\omega_{b} \int_{0}^{1} \xi^{\tilde{C}_{2}}(1+\xi)^{-1 / 2}\left(\tilde{B}_{1}+\eta_{b} \frac{\bar{\gamma}^{2}}{2}+\tilde{B}_{2} \xi^{2}\right)^{-\left(\frac{\tilde{C}_{1}+\tilde{C}_{2}+2}{2}\right)} d \xi\right]
\end{aligned}
$$

$$
P_{2}^{>}(2) \cong \frac{\tilde{A}_{1} \tilde{A}_{2}}{2 \sqrt{2}} \Gamma\left(\frac{\tilde{C}_{1}+\tilde{C}_{2}+2}{2}\right) \sum_{b=1}^{2}\left[\omega_{b} \int_{0}^{1} \xi^{\tilde{C}_{2}}(1+\xi)^{-1 / 2}\left(\tilde{B}_{1}+\eta_{b} \frac{\bar{\gamma}^{2}(1-\xi)^{2}}{8}+\tilde{B}_{2} \xi^{2}\right)^{-\left(\frac{\tilde{C}_{1}+\tilde{C}_{2}+2}{2}\right)} d \xi\right]
$$


[15] H. Jafarkhani and V. Tarokh, "Multiple transmit antenna differential detection from generalized orthogonal designs," IEEE Trans. Inf. Theory, vol. 47, no. 6, pp. 2626-2631, Sep. 2001.

[16] J. Mietzner, R. Schober, L. Lampe, W. H. Gerstacker, and P. A. Hoeher, "Multiple-antenna techniques for wireless communications-a comprehensive literature survey," IEEE Commun. Surveys Tutorials, vol. 11, no. 2, pp. 87-105, 2009.

[17] H. L. Van Trees, Detection, Estimation, and Modulation Theory, Part I. John Wiley \& Sons, Inc. 2001.

[18] J. I. Marcum, "A statistical theory of target detection by pulsed radar," IRE Trans. Inf. Theory, vol. 6, no. 2, pp. 59-267, Apr. 1960.

[19] G. E. Corazza and G. Ferrari, "New bounds for the Marcum Qfunction," IEEE Trans. Inf. Theory, vol. 48, no. 11, pp. 3003-3008, Nov. 2002.

[20] M. Abramowitz and I. A. Stegun, Handbook of Mathematical Functions with Formulas, Graphs, and Mathematical Tables, 9th edition. New York: Dover, 1972.

[21] A. P. Prudnikov, Y. A. Brychkov, and O. I. Marichev, Integrals and Series. Vol. 3: More Special Functions, 2003.

[22] M. Nakagami, "The $m$-distribution: a general formula of intensity distribution of rapid fading," Statistical Methods in Radio Wave Propagation, W. G. Hoffman, editor. Oxford, U.K.: Permagon Press, pp. 3-36, 1960

[23] D. Tse and P. Viswanath, Fundamentals of Wireless Communication. Cambridge University Press, 2005.

[24] M. K. Simon and M.-S. Alouini, Digital Communication over Fading Channels: A Unified Approach to Performance Analysis, 1st edition. John Wiley \& Sons, Inc., 2000.

[25] R. A. Amaral de Souza and M. D. Yacoub, "Bivariate Nakagami- $m$ distribution with arbitrary correlation and fading parameters," IEEE Trans. Wireless Commun., vol. 7, no. 12, pp. 5227-5232, Dec. 2008.

[26] M. Di Renzo and H. Haas, "Spatial modulation with partial-CSI at the receiver: optimal detector and performance evaluation," in Proc. IEEE Sarnoff Symposium, Apr. 2010, pp. 1-6.

[27] M. K. Simon and M.-S. Alouini, "A unified approach to the performance analysis of digital communications over generalized fading channels," Proc. IEEE, vol. 86, no. 9, pp. 1860-1877, Sep. 1998.

[28] G. Ferrari and G. E. Corazza, "Tight bounds and accurate approximations for DQPSK transmission bit error rate," IEEE Electron. Lett., vol. 40, no. 20, pp. 1284-1285, Sep. 2004.

[29] I. S. Gradshteyn and I. M. Ryzhik, Table of Integrals, Series, and Products, 5th edition. Academic Press, Jan. 1994.

[30] G. H. Golub and J. H. Welsch, "Calculation of Gauss quadrature rules," Math. Comput., vol. 23, no. 106, pp. 221-230, Apr. 1969.

[31] M. Chiani, D. Dardari, and M. K. Simon, "New exponential bounds and approximations for the computation of error probability in fading channels," IEEE Trans. Wireless Commun., vol. 2, no. 4, pp. 840-845, July 2003.

[32] J. Reig, M. A. Martinez-Amoraga, and L. Rubio, "Generation of bivariate Nakagami- $m$ fading envelopes with arbitrary not necessary identical fading parameters," Wireless Commun. Mobile Computing, vol. 7, no. 4, pp. 531-537, May 2007.

[33] Q. T. Zhang, "A decomposition technique for efficient generation of correlated Nakagami fading channels," IEEE J. Sel. Areas Commun., vol. 18 , no. 11 , pp. 2385-2392, Nov. 2000.

[34] M. Di Renzo and H. Haas, "Improving the performance of space shift keying (SSK) modulation via opportunistic power allocation," IEEE Commun. Lett., vol. 14, no. 6, pp. 500-502, June 2010.

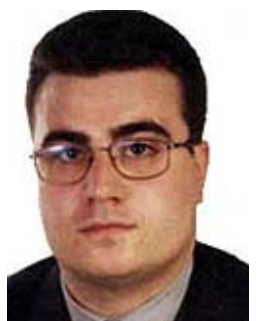

Marco Di Renzo (S'05-AM'07-M'09) was born in L'Aquila, Italy, in 1978. He received the Laurea (cum laude) and the Ph.D. degrees in electrical and information engineering from the Department of Electrical and Information Engineering, University of L'Aquila, Italy, in April 2003 and January 2007, respectively.

From August 2002 to January 2008, he was with the Center of Excellence for Research DEWS University of L'Aquila, Italy. From February 2008 to April 2009, he was a Research Associate with the Telecommunications Technological Center of Catalonia (CTTC), Barcelona, Spain. From May 2009 to December 2009, he was a Research Fellow with the Institute for Digital Communications (IDCOM), The University of Edinburgh, Edinburgh, Scotland, United Kingdom (UK).

Since January 2010, he has been a Researcher ("Chargé de Recherche") with the French National Center for Scientific Research (CNRS), and a research staff member of the Laboratory of Signals and Systems (LSS), a joint research laboratory of the CNRS, the École Supérieure d'Électricité (SUPÉLEC), and the University of Paris-Sud XI, Paris, France. His main research interests are in the area of wireless communication theory, signal processing, and information theory.

In December 2004, he co-founded WEST Aquila s.r.l. (Wireless Embedded Systems Technologies L'Aquila), an R\&D Spin-Off of the Department of Electrical and Information Engineering, and the Center of Excellence for Research DEWS, where he currently holds the position of Senior Research Engineer in wireless communications. In 2006, he was a Visiting Scholar with the Mobile and Portable Radio Research Group (MPRG), in the Bradley Department of Electrical and Computer Engineering, Virginia Polytechnic Institute and State University, USA.

Dr. Di Renzo was awarded a special mention for his outstanding fiveyear (1997-2003) academic career from the University of L'Aquila, Italy; a three-year Ph.D. fellowship (ranked 1st) from the Department of Electrical and Information Engineering, University of L'Aquila, Italy, and THALES Communications s.p.a, Land and Joint Systems Division of Advanced Studies, Chieti, Italy; and a personal "Torres Quevedo" Grant (PTQ-08-01-06437) from the "Ministry of Science and Innovation" in Spain for his research on ultra wide band wireless systems and cooperative localization for wireless sensor networks.

Dr. Di Renzo is a Member of the IEEE and IEEE Communications Society, and serves as a reviewer for transaction journals and international conferences. He served as Publicity Chair of the 2010 International Conference on Mobile Lightweight Wireless Systems (Mobilight). He also serves as Technical Program Committee (TPC) member and Session Chairman of several international conferences in communications.

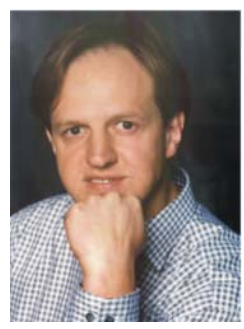

Harald Haas (S'98-AM'00-M'03) received the $\mathrm{Ph} . \mathrm{D}$. degree from the University of Edinburgh in 2001. His main research interests are in the areas of wireless system design/analysis and digital signal processing, with a particular focus on interference aware MAC protocols, multiuser access, link adaptation, scheduling, dynamic resource allocation, multiple antenna systems, and optical wireless communication.

$\mathrm{He}$ joined the International University Bremen (Germany), now Jacobs University Bremen, in September 2002, where he was Professor of Electrical Engineering. In June 2007, he joined the University of Edinburgh (Scotland/UK), where he is currently Professor of Mobile Communications in the Institute for Digital Communications (IDCOM).

Haas received a best paper award at the International Symposium on Personal, Indoor and Mobile Radio Communications (PIMRC) in Osaka/Japan in 1999, and holds more than 15 patents in the area of wireless communications. Haas contributed a chapter to the Handbook of Information Security entitled "Air Interface Requirements for Mobile Data Services" by John Wiley \& Sons, Inc. He co-authored a book entitled Next Generation Mobile Access Technologies: Implementing TDD with Cambridge University Press. His work on optical wireless communication was selected for publication in 100 Produkte der Zukunft (100 Products of the Future) authored by Nobel Laureate T. W. Hänsch. Since 2007, Haas is a Regular High Level Visiting Scientist supported by the Chinese "111 program" at Beijing University of Posts and Telecommunications. 